\title{
Gpnmb inhibits oligodendrocyte differentiation of adult neural stem cells by amplifying TGF $\beta 1$ signaling
}

\author{
Daniel Z. Radecki ${ }^{1,2}$, Albert R. Wang ${ }^{3,4,5}$, Abigail S. Johnson ${ }^{1,2}$, Christian A. \\ Overman $^{1,2}$, Madison M. Thatcher ${ }^{1,2}$, Gopal lyer ${ }^{3,4}$, Jayshree Samanta ${ }^{1,2,6^{*}}$ \\ ${ }^{1}$ Department of Comparative Biosciences, School of Veterinary Medicine, University of \\ Wisconsin-Madison, Madison, WI 53706, USA \\ ${ }^{2}$ Stem Cell and Regenerative Medicine Center, University of Wisconsin-Madison, \\ Madison, WI 53705, USA \\ ${ }^{3}$ Department of Human Oncology, School of Medicine and Public Health, University of \\ Wisconsin, 600 Highland Avenue, K4/B100-0600, Madison, WI, 53792, USA. \\ ${ }^{4}$ University of Wisconsin Carbone Cancer Center, School of Medicine and Public Health, \\ University of Wisconsin, Madison, WI, USA. \\ ${ }^{5}$ Department of Biomedical Engineering, University of Wisconsin-Madison, Madison, WI \\ 53706, USA \\ ${ }^{6}$ Lead contact \\ *Correspondence: Jayshree.samanta@wisc.edu
}




\section{Summary}

Gli1 expressing neural stem cells, in the subventricular zone of the adult mammalian brain, respond to demyelination injury by differentiating into oligodendrocytes. We have identified Gpnmb as a novel regulator of oligodendrogenesis in Gli1 neural stem cells, whose expression is induced by TGF $\beta 1$ signaling via Gli1, in response to a demyelinating injury. Upregulation of Gpnmb further activates the TGF $\beta 1$ pathway by increasing the expression of the TGF $\beta 1$ binding receptor subunit, TGF $\beta R 2$. Thus the TGF $1 \rightarrow$ Gli1 $\rightarrow$ Gpnmb $\rightarrow$ TGF $\beta$ R2 signaling pathway forms a feed forward loop for sustained activation of TGF $\beta 1$ signaling in Gli1 neural stem cells, resulting in inhibition of their differentiation into mature oligodendrocytes following demyelination.

\section{Keywords}

Neural stem cell, Oligodendrocyte, Demyelination, Remyelination, TGF $\beta 1$, Gpnmb, Gli1, Subventricular zone 


\section{INTRODUCTION}

The adult subventricular zone (SVZ) consists of quiescent neural stem cells that are multipotent, divide slowly and self-renew (Doetsch et al., 1999). There is considerable heterogeneity amongst these cells, indicated by their expression of specific transcription factors and generation of distinct cells in homeostasis and injury (Chaker et al., 2016). One of the subsets residing in the ventral SVZ accounts for $25 \%$ of the quiescent neural stem cells, expresses the transcription factor Gli1, and generates interneurons in the olfactory bulb and astrocytes in the healthy mouse forebrain (Ahn and Joyner, 2005). These ventral neural stem cells (vNSCs) are also present in the human SVZ (Samanta et al., 2015). Remarkably, the Gli1 vNSCs can be recruited to the white matter where they differentiate into myelinating oligodendrocytes in response to demyelination but not in the healthy adult brain (Samanta et al., 2015; Sanchez and Armstrong, 2018; Sanchez et al., 2018). Inhibition of Gli1 in these cells further enhances their remyelination potential and is neuroprotective in mouse models of multiple sclerosis (MS) (Samanta et al., 2015). In this study we analyzed the transcriptome of VNSCs to discover that repression of TGF 1 signaling pathway along with downregulation of glycoprotein non-metastatic melanoma b (Gpnmb) are accompanied by loss of Gli1 in these cells, following demyelination.

The TGF $\beta$ family influences proliferation, migration and differentiation of NSCs, along with modulating the immune system (David and Massague, 2018). Indeed, TGF $\beta$ ligands including TGF $\beta 1$ as well as the receptor subunits TGF $\beta R 1$ and TGF $\beta R 2$ are highly expressed by reactive astrocytes and microglia in chronic MS lesions (De Groot et al., 1999). TGF $\beta 1$ is also upregulated in the aging brain where remyelination is limited (Nicaise et al., 2019; Pasinetti et al., 1999). In rodents, demyelinating lesions show higher TGFß1 expression (Hinks and Franklin, 2000) and transgenic overexpression of TGF $\beta 1$ in the brain results in earlier onset and more severe disease in the EAE model suggesting a 
negative role in remyelination (Wyss-Coray et al., 1997). On the contrary, genetic knockout of TGF $\beta 1$ in the developing embryo results in focal demyelination in the brain suggesting a positive effect on developmental myelination (Makwana et al., 2007). Thus, the actions of TGF $\beta 1$ signaling are dependent on the timing, concentration and cell specific modulators of the pathway. TGF $\beta 1$ signals by binding to the receptor TGF $\beta$ R2 leading to recruitment and phosphorylation of TGF $\beta R 1$ receptor thus activating its kinase. Activated TGF $\beta R 1$ phosphorylates Smad2/3, which form a complex with Smad4 and translocate to the nucleus for regulation of transcription (Massague, 1987; Wrana et al., 1994).

In this study, we identified a novel regulator of remyelination - Gpnmb, via its role in activation of the TGF $\beta 1$ pathway by upregulating TGF $\beta$ R2 expression in vNSCs. Gpnmb is a single-pass transmembrane glycoprotein with a C-terminus cytoplasmic domain and an N-terminus extracellular domain. The human and mouse genes are very similar with a high degree of conservation in the chromosomal region around Gpnmb in both genomes (Owen et al., 2003). Gpnmb is upregulated in many neurodegenerative diseases featuring demyelination such as in amyotrophic lateral sclerosis, Alzheimer's disease, Parkinson's disease and MS (Hendrickx et al., 2017; Hüttenrauch et al., 2018; Moloney et al., 2018; Satoh et al., 2019; Tanaka et al., 2012). In MS brains, Gpnmb is highly expressed in chronic active lesions, which are associated with more aggressive disease and declining remyelination (Hendrickx et al., 2017).

In this study, we have used a Gpnmb-LacZ knock-in mouse to not only characterize the previously unknown expression of Gpnmb in the adult forebrain but also to elucidate its role in regulation of remyelination by vNSCs. Our results indicate that demyelination induces TGF $\beta 1$, which acts via Gli1 to upregulate Gpnmb in vNSCs; overexpression of Gpnmb further induces expression of the TGF $\beta$ R2 receptor thus 
enhancing the activation of the TGF $\beta 1$ pathway, leading to the reduced generation of mature OLs from vNSCs.

\section{RESULTS}

\section{Identification of Gpnmb as a novel inhibitor of oligodendrogenesis in ventral neural stem cells}

Our previous studies have shown that loss of Gli1 in vNSCs of the adult SVZ, results in enhanced recruitment and regeneration of myelinating oligodendrocytes in response to demyelination of the white matter corpus callosum (CC) (Radecki et al., 2020; Samanta et al., 2015). To determine the molecular mechanisms involved, we compared the transcriptomes of vNSCs isolated from the healthy and demyelinated brains of the

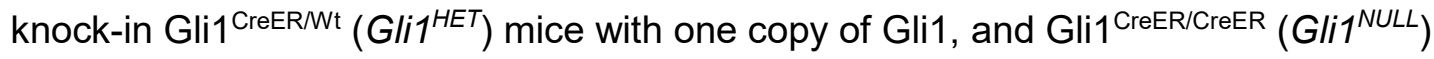
mice with loss of Gli1 expression (Samanta, 2021). We probed the top 100 differentially

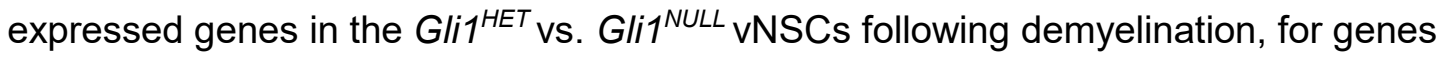
shared amongst three or more GO terms related to cell survival, migration and differentiation. Through this analysis, we identified Gpnmb, which was also the most significantly downregulated gene, with 7.9 fold lower expression, in Gli1 ${ }^{N U L L}$ VNSCs, suggestive of its role as a negative regulator of remyelination (Fig.1A). Notably, Gpnmb was upregulated following demyelination in Gli $1^{\text {HET }}$ vNSCs but not in the Gli ${ }^{\text {NULL }}$ vNSCs (Fig.1A), further suggesting that lack of Gpnmb induction in Gli1 ${ }^{N U L L}$ vNSCs may be beneficial for remyelination.

To determine if Gpnmb plays a role in remyelination, we examined the consequences of genetic loss of Gpnmb using a $\beta$-Galactosidase knock-in Gpnmb ${ }^{\text {Lacz }}$ mouse (Table S1), thus allowing us to identify the cells with an active Gpnmb promoter

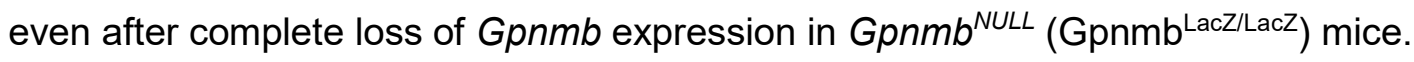
Global loss of Gpnmb did not result in any overt phenotype and the mice were healthy 
and fertile with a normal lifespan. We induced demyelination in the Gpnmb ${ }^{\text {NULL }}$

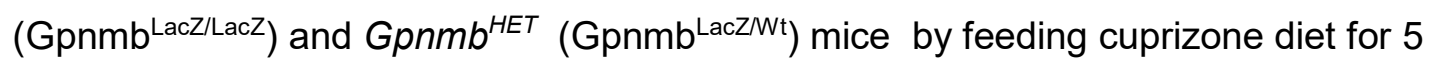
weeks and analyzed the brains at 2 weeks of recovery from the diet (Fig.1B). In this model, cuprizone diet results in peak demyelination at 5-6 weeks and returning the mice to regular chow allows remyelination to occur spontaneously (Matsushima and Morell, 2001). While the extent of demyelination was similar in Gpnmb $b^{N U L L}$ and Gpnmb ${ }^{W T}$ mice (Fig. S1A), the number of $\mathrm{CC} 1$-expressing mature oligodendrocytes in the $\mathrm{CC}$ of $G p n m b^{N U L L}$ mice was $2.7 \pm 0.1$ fold higher than in the $G p n m b^{W T}$ and $1.8 \pm 0.4$ fold higher than Gpnmb $b^{H E T}$ mice (Fig.1 C,D). However, the number of NG2+ oligodendrocyte progenitor cells (OPCs) did not change upon loss of Gpnmb (Fig.1C,E). Taken together these results indicate that Gpnmb inhibits oligodendroglial differentiation in the white matter following demyelination.

To determine if the increase in OLs in the CC following demyelination was due to an increase in recruitment and/or differentiation of vNSCs, we traced the lineage of Gli1 vNSCs by genetically fate-mapping the cells in the Gpnmb $b^{N U L L}$ mice at 2 weeks of recovery from cuprizone diet, using Gli1 ${ }^{H E T} ;$ Gpnmb $b^{\text {NULL }}$ mice (Table S1). Consistently, we found a significant $2.7 \pm 0.1$ fold increase in the proportion of CC1+RFP double positive cells derived from fate-mapped vNSCs in the Gli1 ${ }^{\text {HET }}$;Gpnmb ${ }^{\text {NULL }}$ CC compared to the Gli1 ${ }^{H E T}$;Gpnmb $b^{\text {HET }} \mathrm{CC}$, indicating an increase in generation of mature OLs from the Gli1 vNSCs $(F i g .1 F, G)$. However, there was no change in the number of NG2+RFP double positive OPCs or GFAP+RFP double positive astrocytes derived from fate-mapped VNSCs between the Gli1 ${ }^{\text {HET }}$;Gpnmb $b^{\text {HET }}$ and Gli1 ${ }^{\text {HET }}$;Gpnmb $b^{\text {NULL }}$ mice (Fig.1F,G; Fig.S1B). In addition, the progeny of Gli1 vNSC cells did not differentiate into NeuN+ neurons or Iba1+ microglia (Fig.S1B). Thus, the Gli1 vNSCs generate more OLs without affecting their differentiation into OPCs, astrocytes, neurons or microglia. To examine if the increase in OLs was in part due to an increase in the number of Gli1 vNSCs recruited to 
the CC, we quantified the total number of RFP+ fate-mapped Gli1 vNSCs in the CC, but did not observe a significant change in the Gli1 ${ }^{H E T}$;Gpnmb $b^{N U L L}$ mice compared to the Gli1 ${ }^{H E T}$; Gpnmb ${ }^{H E T}$ mice (Fig.1F,H). In addition, there was no change in the number of fate-mapped RFP+ vNSCs with an active Gpnmb promoter (LacZ+), between the Gli1 ${ }^{H E T} ;$ Gpnmb $b^{H E T}$ and Gli1 ${ }^{H E T} ;$ Gpnmb $b^{\text {NULL }}$ mice (Fig.S1C-D). These data indicate that loss of Gpnmb increases the differentiation of cells derived from Gli1 vNSC into OLs, in the white matter following demyelination.

\section{Expression of Gpnmb is limited to NSCs and glial cells in the forebrain}

To characterize the expression of Gpnmb in the quiescent NSCs, we first quantified the number of LacZ+ cells co-expressing GFAP in the entire SVZ at 1 week of recovery from cuprizone diet as compared to the healthy SVZ in Gpnmb ${ }^{H E T}$ mice, but did not observe any difference between the healthy and remyelinating SVZ (Fig.2A-B). In addition there were no differences in the proportion of LacZ+ cells co-expressing markers of OLs (CC1), OPCs (PGFRa), neurons (NeuN) and microglia (lba1) in the healthy vs. remyelinating SVZ (Fig.2B). To examine the expression of Gpnmb specifically in Gli1 vNSCs, we quantified the RFP+ fate-mapped Gli1 cells co-expressing LacZ in the Gli1 ${ }^{H E T} ;$ Gpnmb $b^{\text {HET }}$ mice. There was a significant $1.5 \pm 0.1$ fold increase in the proportion of RFP+LacZ double positive cells in the SVZ, at 1 week of recovery from cuprizone diet $(45.9 \pm 2.6 \%)$ compared to the healthy SVZ (31.3 $\pm 3.9 \%)$ (Fig.2C-D), consistent with the RNAseq data showing increase in Gpnmb expression upon demyelination in the Gli ${ }^{H E T}$ NSCs.

Loss of Gpnmb can potentially enhance remyelination due to its effects on OPCs and OLs. In addition, several RNAseq studies have indicated that Gpnmb is expressed in oligodendroglial and microglial cells in the mouse and human brain (Srinivasan et al., 2020; Zhang et al., 2014; Zhang et al., 2016). Therefore, we characterized the 
expression of Gpnmb in the CC and cortex of the healthy adult Gpnmb ${ }^{H E T}$ forebrain and following 1 week of recovery from cuprizone induced demyelination. In the healthy CC, the Gpnmb expressing cells consisted of GFAP+ astrocytes (10.3 $\pm 1.3 \%)$, Iba1+

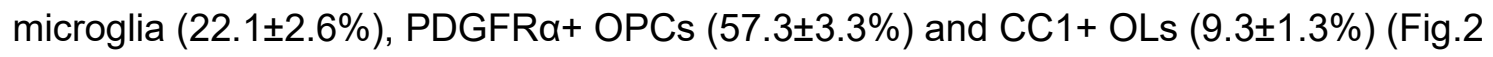
E-I). However, at 1 wk of recovery from cuprizone diet, Gpnmb expressing GFAP+ astrocytes increased $(4.7 \pm 0.3$ fold $)$ with a concomitant significant reduction in Iba1+

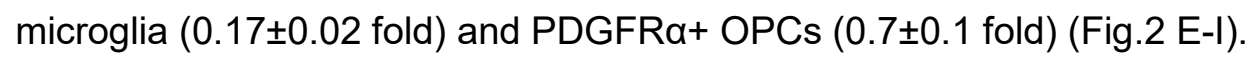

In the healthy cortex, the Gpnmb expressing cells were comprised of $1.8 \pm 0.2 \%$ GFAP+ astrocytes, $20.2 \pm 3.7 \%$ Iba1+ microglia, 74.6 $\pm 4.4 \%$ PDGFR $\square+$ OPCs and $5.6 \pm 0.6 \% \mathrm{CC} 1+$ OLs (Fig.2 E-J). Compared to the healthy CC, the cortex had $1.3 \pm 0.1$ fold higher number of Gpnmb+ OPCs and a 0.18 \pm 0.02 fold lower number of Gpnmb+ astrocytes. Similar to the CC, Gpnmb expression significantly increased by $13.2 \pm 1.8$ fold in astrocytes and decreased by $0.07 \pm 0.02$ fold in microglia, in the cortex at 1 week of recovery from cuprizone diet (Fig.2 E-J). Notably, Gpnmb was not expressed in neurons in the healthy or remyelinating SVZ, CC, and cortex.

Thus, Gpnmb is expressed in astroglial, oligodendroglial and microglial cells along with NSCs in the healthy forebrain and its expression is dynamically regulated by demyelinating injury.

\section{Gpnmb is necessary for inhibition of oligodendrogenesis by TGF $\beta 1$}

By performing an Ingenuity and Kegg pathway analyses of the RNAseq data (Samanta, 2021), we identified the TGF 1 pathway as significantly activated in Gli1 ${ }^{H E T}$ vNSCs as compared to Gli1 ${ }^{N U L L}$ vNSCs following demyelination (Fig.3A). To confirm the source of TGF $\beta 1$ in the adult brain, we compared its mRNA expression in the CC, cortex and SVZ of healthy vs demyelinated brain. After 3 weeks of cuprizone diet, the timepoint which coincides with the initiation of demyelination, Tgfb1 expression showed a 
significant 2.2 \pm 0.4 fold increase in the $\mathrm{CC}$, but remained unchanged in the cortex and SVZ (Fig.3B). This suggests that TGF $\beta 1$ is secreted from the lesion in response to a demyelinating injury. To examine if TGF $\beta 1$ can regulate Gpnmb expression, we harvested vNSCs from the SVZ of adult Gli1 ${ }^{H E T}$ and Gli1 ${ }^{\text {NULL }}$ mice and grew them as neurospheres in vitro, followed by differentiation of the dissociated neurospheres in adherent cultures for 14 days (Fig.3C). Treatment of vNSCs with TGF $\beta 1$ (10ng/mL), significantly increased Gpnmb mRNA levels by $4.3 \pm 1.2$ fold in Gli1 ${ }^{H E T}$ VNSCs, but not in Gli1 ${ }^{\text {NULL }}$ vNSCs (Fig. 3D). Moreover this effect on Gpnmb upregulation was very specific to TGF $\beta 1$, since treatment with other growth factors like Shh $(100 \mathrm{ng} / \mathrm{mL})$ (Galvin et al., 2008) and BMP-4 (20ng/mL) (Bond et al., 2014; Samanta et al., 2007) failed to change Gpnmb expression in both Gli1 ${ }^{H E T}$ and Gli $1^{\text {NULL }}$ vNSCs (Fig.3D). These results suggest that TGF $\beta 1$ acts via Gli1 to upregulate Gpnmb expression in Gli1 ${ }^{H E T}$ vNSCs, and consequently fails to induce Gpnmb in Gli ${ }^{\text {NULL }}$ vNSCs.

To examine the effect of TGF $\beta 1$ on differentiation of vNSCs, we quantified the proportion of RFP+ cells co-expressing markers of OLs, OPCs, or astrocytes following TGF $\beta 1$ treatment. Although TGF $\beta 1$ did not alter the generation of OLs from Gli1 ${ }^{H E T}$ vNSCs, it significantly increased the differentiation of Gli1 ${ }^{N U L L}$ vNSCs into OLs by 5.2 \pm 1.3 fold (Fig.3 E-F) and decreased their differentiation into astrocytes by $0.5 \pm 0.1$ fold (Fig.S2 A-B). Consistent with previous results there was no change in the differentiation of Gli ${ }^{H E T}$ and Gli1 ${ }^{\text {NULL }}$ vNSCs into OPCs (Fig.3G,H). Thus, TGF $\beta 1$ treatment upregulates Gpnmb but does not affect the generation of OLs from Gli1 ${ }^{H E T}$ vNSCs. In contrast, it increases the differentiation of Gli1 ${ }^{N U L L}$ vNSCs into OLs, without affecting Gpnmb expression. This suggests that upregulation of Gpnmb by TGF $\beta 1$, inhibits the differentiation of vNSCs into OLs.

To directly examine the inhibitory role of Gpnmb in oligodendrogenesis, we overexpressed Gpnmb with lentiviral transduction of the Gpnmb cDNA fused with GFP 
(Gpnmb-OE), which significantly increased Gpnmb mRNA levels by $402 \pm 141$ fold in vNSCs (Fig.S3A). The efficiency of lentiviral infection was similar in both Gli $1^{H E T}$ and Gli1 ${ }^{\text {NULL }}$ vNSCs (Fig.S3B). We then quantified the number of OLs, OPCs and astrocytes generated from Gli1 ${ }^{H E T}$ and Gli1 ${ }^{\text {NULL }}$ vNSCs overexpressing Gpnmb i.e. proportion of RFP+GFP double positive cells co-expressing the cell type specific markers. Gpnmb overexpression significantly decreased the generation of OLs by $3.8 \pm 0.6$ fold in Gli $1^{H E T}$ and 3.5 \pm 0.3 fold in Gli1 ${ }^{\text {NULL }}$ vNSCs (Fig. $\left.3 \mathrm{I}, \mathrm{J}\right)$. However it did not alter the differentiation of vNSCs into OPCs (Fig.S3 C,D) and astrocytes (Fig.S3 E,F).

Together, these results indicate that demyelination increases TGF $\beta 1$ expression in the lesion, which induces Gpnmb expression via Gli1, leading to reduction in generation of mature OLs from vNSCs.

\section{Gpnmb inhibits oligodendrogenesis by inducing TGF $\beta$ R2 expression}

Since the RNAseq data (Samanta, 2021) showed significantly higher expression of the ligand binding subunit of TGF $\beta$ receptor, TGF $\beta R 2$, in the Gli1 ${ }^{H E T}$ VNSCs compared to the Gli1 ${ }^{\text {NULL }}$ vNSCs upon demyelination (Fig.3 A), it suggested that Gpnmb can regulate the TGF $\beta 1$ pathway. Consistently, overexpression of Gpnmb in wildtype vNSCs in vitro, resulted in a significant upregulation of Tgfbr2 by $2.2 \pm 0.5$ fold but did not change Tgfbr1 subunit mRNA expression (Fig.4A), indicating a potential feed forward loop consisting of TGF $\beta 1 \rightarrow$ Gli1 $\rightarrow$ Gpnmb $\rightarrow$ TGF $\beta$ R2. To confirm this, we examined Tgfbr2 expression in the SVZ of Gpnmb $b^{\text {HET }}$ and Gpnmb $b^{\text {NULL }}$ mice. Consistent with the data from vNSCs, in vivo loss of Gpnmb also resulted in a significant reduction in Tgfbr2 expression by $0.54 \pm 0.05$ fold without altering Tgfbr 1 expression and this effect was not due to loss of Tgfb1 expression in Gpnmb ${ }^{N U L L}$ mice (Fig.4B). Thus TGF $\beta 1$ induces Gpnmb via Gli1, which in turn induces TGF $\beta R 2$. 
Next, we wanted to confirm whether loss of TGFßR2 was sufficient to alter vNSC recruitment or differentiation in vivo. We conditionally knocked out Tgfbr2 from Gli1 ${ }^{H E T}$ and Gli ${ }^{\text {NULL }}$ VNSCs and examined the fate of their progeny after inducing demyelination by stereotactic injection of Lysophosphatidyl choline (LPC) (Plemel et al., 2018) (Fig.4 C). We confirmed the ablation of TGF $\beta 1$ signaling in Gli1 vNSCs, by the absence of pSmad3, a downstream effector of TGF 1 signaling pathway (Massague, 1987; Wrana et al., 1994) (Fig.S4A). In addition, we confirmed that the extent of demyelination induced by LPC injection was similar in both $T g f b r 2^{H E T}$ and $T g f b r 2^{N U L L}$ mice (Fig.S4B).

We then analyzed the recruitment of the Gli1 ${ }^{H E T}$ and Gli $1^{\text {NULL }}$ vNSCs to the CC, 1 week after LPC or vehicle injection. The total number of RFP+ cells in the lesion, was significantly higher by $6.6 \pm 1.1$ fold in Gli1 ${ }^{H E T} ; \operatorname{Tgfbr}^{N U L L}, 5.4 \pm 0.4$ fold in

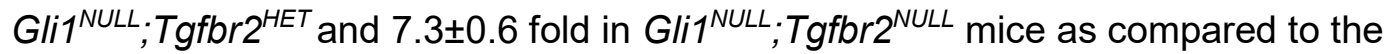
respective vehicle injected controls (Fig.4D,E). Thus, while complete loss of Tgfbr2 increased the recruitment of both Gli1 ${ }^{\text {HET }}$ and Gli1 ${ }^{\text {NULL }}$ vNSCs, loss of one copy of Tgfbr2 was sufficient to increase the recruitment of Gli1 ${ }^{N U L L}$ vNSCs, with the highest recruitment observed in mice with loss of both Gli1 and Tgfbr2.

To examine the fate of the recruited cells in the CC, we quantified the RFP+ cells co-expressing markers of OLs, OPCs and astrocytes. The number of OPCs generated from RFP+ cells did not alter with loss of Tgfbr2 in both Gli $1^{\mathrm{HET}}$ and Gli $1^{\mathrm{NULL}}$ vNSCs (Fig.4 F). However the proportion of OLs derived from RFP+ cells significantly increased with loss of both Gli1 and Tgfbr2 in Gli $1^{\text {NULL }} ; T_{\text {Tfbr }}{ }^{\text {NULL }}$ mice, with $2.54 \pm 0.08$ fold higher OLs than Gli1 ${ }^{H E T} ; T G F \beta R 2^{H E T}$ mice (Fig.4G).

Taken together, these results not only indicate the synergistic effects of loss of Gli1 and TGF $\beta 1$ signaling in vNSCs, but also the role of Gpnmb in amplifying TGF $\beta 1$ signaling by inducing Tgfbr2 leading to inhibition of recruitment and their differentiation into mature OLs. 


\section{Discussion}

Our results show that demyelination increases the levels of TGF $\beta 1$, leading to upregulation of Gpnmb expression which further activates the pathway by inducing TGFßR2 in vNSCs, ultimately blocking their differentiation into oligodendrocytes. Thus, Gpnmb is a downstream effector of TGF $\beta 1$ signaling in vNSCs and functions in a feed forward manner to sustain and amplify the pathway, resulting in inhibition of remyelination.

TGF $\beta 1$ expression is very low in the healthy brain, but is highly induced in response to injury and neurodegenerative diseases like Alzheimer's and Parkinson's disease, amyotrophic lateral sclerosis, stroke, traumatic brain injury and MS (Finch et al., 1993; Flanders et al., 1995; Ilzecka et al., 2002; Issazadeh et al., 1995; Krupinski et al., 1996; Lindholm et al., 1992; Mogi et al., 1995; Rimaniol et al., 1995). Likewise, our results also show that it is upregulated in the white matter $\mathrm{CC}$ following demyelination (Fig.3B) and induces Gpnmb in Gli $1^{\text {HET }}$ vNSCs but not in Gli1 ${ }^{\text {NULL }}$ vNSCs (Fig.3D). Indeed, Gpnmb expression was significantly lower in Gli $1^{\text {NULL }}$ VNSCs compared to Gli $1^{\mathrm{HET}}$ vNSCs following demyelination (Fig.1A), although there was no difference in Gpnmb levels in the healthy brain (Samanta, 2021). This indicates that Gli1 is necessary for upregulation of Gpnmb following demyelination but not required for its baseline expression in healthy brains. Since TGF $\beta 1$ is known to increase Gli1 expression in various other tissues (Dennler et al., 2007), the upregulation of Gpnmb in response to demyelination is likely due to the induction of Gli1 by TGF $\beta 1$ and therefore cannot be upregulated in Gli1 ${ }^{\text {NULL }}$ vNSCs.

Similar to TGF $\beta 1$ expression, Gpnmb is also highly expressed in many neurodegenerative diseases like amyotrophic lateral sclerosis, Alzheimer's disease, Parkinson's disease and MS (Hendrickx et al., 2017; Hüttenrauch et al., 2018; Moloney et al., 2018; Satoh et al., 2019; Tanaka et al., 2012). In addition to neural stem cells, Gpnmb is expressed in astrocytes, microglia, OLs and OPCs in the SVZ, CC and cortex of the 
healthy adult forebrain (Fig.2). However, Gpnmb is not expressed in neurons of the forebrain. In response to demyelination, the proportion of Gli1 vNSCs co-expressing Gpnmb increased significantly in the SVZ along with an increase in astrocytic expression in the CC and cortex (Fig.2), consistent with its induction by TGF $\beta 1$. Furthermore, global loss of Gpnmb significantly increased the mature OLs derived from Gli1 vNSCs without affecting the number of OPCs (Fig.1F-G), indicating its function in maturation of the oligodendrocyte lineage. Consistently, treatment of vNSCs with TGF $\beta 1$ increased the number of mature OLs, only in Gli1 ${ }^{\text {NULL }}$ vNSCs which are unable to induce Gpnmb in response to TGF $\beta 1$ (Fig.3 E-F). This was further confirmed by directly overexpressing Gpnmb in vNSCs, which inhibited the generation of mature OLs from Gli ${ }^{\text {NULL }}$ vNSCs (Fig.3 I,J).

TGF $\beta 1$ binds to the receptor TGF $\beta R 2$, which is highly enriched in the neural stem cells of the adult SVZ, and inhibits their proliferation without affecting their differentiation fate into neurons, astrocytes and OPCs, but their differentiation into mature OLs had not been examined (Wachs et al., 2006). Our results show that TGF $\beta 1 \rightarrow$ Gli1 $\rightarrow$ Gpnmb signaling inhibits the differentiation into mature OLs. However, the effects of TGF $\beta 1$ are dependent on dosage and time of exposure. Indeed, we found that demyelination leads to sustained activation of TGF $\beta 1$ in vNSCs due to induction of TGF $\beta 22$ by Gpnmb, thus altering the cell fate of vNSCs (Fig.4 A,B). However, conditional ablation of TGF $\beta$ R2 specifically in Gli1 vNSCs, enhanced the recruitment of both Gli ${ }^{\text {HET }}$ and Gli $1^{\text {NULL }}$ vNSC derived cells into the lesion, but increased generation of OLs only from Gli1 ${ }^{\text {NULL }}$ vNSCs (Fig.4E-G). This points towards the presence of other Gpnmb independent mechanisms for expression of TGF $\beta R 2$ in Gli1 ${ }^{\text {NULL }}$ VNSCs, consistent with its expression in the

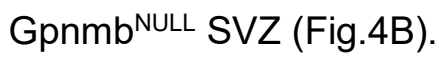

Taken together, these results show that enhanced TGF $\beta 1$ signaling inhibits the recruitment and differentiation of Gli1 vNSCs in demyelinated lesions in part through 
sustained TGF $\beta 1$ activation with a TGF $\beta 1 \rightarrow$ Gli1 $\rightarrow$ Gpnmb $\rightarrow$ TGF $\beta$ R2 feedforward loop. Identifying the cellular mediators for recruitment and differentiation in vNSCs, may ultimately provide therapeutic targets for enhancing repair and regeneration in neurodegenerative diseases.

\section{Limitations of study}

Although we have focused on TGF $\beta 1$ because of its relevance to aging and neurodegenerative diseases, there may be other signaling pathways that regulate Gpnmb in vNSCs. We have studied the effects of global loss of Gpnmb on Gli1 vNSCs, but Gpnmb is also expressed in OPCs, astrocytes and microglia. The cell autonomous effects of Gpnmb in each cell type may be different from those observed in vNSCs. Gpnmb is a transmembrane protein that is cleaved by proteases into an intracellular domain (Gpnmb-ICD) and an ectodomain (Gpnmb-ECD). While Gpnmb-ICD can signal intrinsically by translocating to the nucleus (Utsunomiya et al., 2012), the released Gpnmb-ECD functions as an autocrine or paracrine signal by interacting with CD44 receptor in the same cell or a neighboring cell, respectively (Neal et al., 2018). In this study, we have examined the effects of complete loss of signaling via Gpnmb-ICD and Gpnmb-ECD on recruitment and differentiation of vNSCs in response to demyelination. Further studies are, therefore, needed to differentiate the cell-intrinsic signaling via Gpnmb-ICD vs. extrinsic signaling via secreted Gpnmb-ECD on remyelination.

\section{Acknowledgements}

We thank A.R.W. and G.I. for assistance with bioinformatics analysis as well as the generation of Fig.1A and 3A. A.S.J. and M.M.T. for assistance with experiments in Fig.2, and C.A.O. with assistance with mouse colony maintenance, breeding, and cryosectioning. D.Z.R. was supported in part by a postdoctoral fellowship from the Stem Cell and Regenerative Medicine Center (SCRMC) at UW-Madison. J.S. was funded by the Marie and Jean-Pierre Boespflug Foundation for Myopathic Research and by the 
University of Wisconsin - Madison Office of the Vice Chancellor for Research and

Graduate Education with funding from the Wisconsin Alumni Research Foundation.

\section{Author Contributions}

Conceptualization, D.Z.R. and J.S.; Methodology, D.Z.R. and J.S.; Formal Analysis, A.W., G.I., D.Z.R. and J.S.; Investigation, D.Z.R., A.S.J. and M.M.T.; Resources, D.Z.R., C.A.O., A.W., G.I. and J.S.; Visualization, D.Z.R. and J.S.; Writing - Original Draft, D.Z.R. and J.S.; Writing - Review \& Editing, D.Z.R., A.W., A.S.J., M.M.T., C.A.O, G.I. and J.S.; Funding Acquisition, J.S. and D.Z.R.; Supervision, J.S.

\section{Declaration of Interests}

A patent on the method of targeting GLI1 as a strategy to promote remyelination has been awarded, with J.S. listed as a co-inventor. 


\section{Reference List}

Ahn, S., and Joyner, A.L. (2005). In vivo analysis of quiescent adult neural stem cells responding to Sonic hedgehog. Nature 437, 894-897.

Ahsan, S., and Drăghici, S. (2017). Identifying Significantly Impacted Pathways and

Putative Mechanisms with iPathwayGuide. Current Protocols in Bioinformatics 57, 7.15.11-17.15.30.

Benjamini, Y., and Hochberg, Y. (1995). Controlling the False Discovery Rate: A Practical and Powerful Approach to Multiple Testing. Journal of the Royal Statistical Society Series B (Methodological) 57, 289-300.

Blighe, K., Rana, S., and Lewis, M. (2020). EnhancedVolcano: Publication-ready volcano plots with enhanced colouring and labeling.

Bond, A.M., Peng, C.-Y., Meyers, E.A., McGuire, T., Ewaleifoh, O., and Kessler, J.A. (2014). BMP Signaling Regulates the Tempo of Adult Hippocampal Progenitor Maturation at Multiple Stages of the Lineage. STEM CELLS 32, 2201-2214.

Chaker, Z., Codega, P., and Doetsch, F. (2016). A mosaic world: puzzles revealed by adult neural stem cell heterogeneity. Wiley Interdiscip Rev Dev Biol 5, 640-658.

David, C.J., and Massague, J. (2018). Contextual determinants of TGFbeta action in development, immunity and cancer. Nat Rev Mol Cell Biol 19, 419-435.

De Groot, C.J., Montagne, L., Barten, A.D., Sminia, P., and Van Der Valk, P. (1999). Expression of transforming growth factor (TGF)-beta1, -beta2, and -beta3 isoforms and TGF-beta type I and type II receptors in multiple sclerosis lesions and human adult astrocyte cultures. J Neuropathol Exp Neurol 58, 174-187.

Dennler, S., Andre, J., Alexaki, I., Li, A., Magnaldo, T., ten Dijke, P., Wang, X.J., Verrecchia, F., and Mauviel, A. (2007). Induction of sonic hedgehog mediators by 
transforming growth factor-beta: Smad3-dependent activation of Gli2 and Gli1 expression in vitro and in vivo. Cancer Res 67, 6981-6986.

Doetsch, F., Caille, I., Lim, D.A., Garcia-Verdugo, J.M., and Alvarez-Buylla, A. (1999). Subventricular zone astrocytes are neural stem cells in the adult mammalian brain. Cell $97,703-716$.

Donato, M., Xu, Z., Tomoiaga, A., Granneman, J.G., Mackenzie, R.G., Bao, R., Than, N.G., Westfall, P.H., Romero, R., and Draghici, S. (2013). Analysis and correction of crosstalk effects in pathway analysis. Genome Res 23, 1885-1893.

Draghici, S., Khatri, P., Tarca, A.L., Amin, K., Done, A., Voichita, C., Georgescu, C., and Romero, R. (2007). A systems biology approach for pathway level analysis. Genome Res 17, 1537-1545.

Finch, C.E., Laping, N.J., Morgan, T.E., Nichols, N.R., and Pasinetti, G.M. (1993). TGFbeta 1 is an organizer of responses to neurodegeneration. J Cell Biochem 53, 314-322.

Flanders, K.C., Lippa, C.F., Smith, T.W., Pollen, D.A., and Sporn, M.B. (1995). Altered expression of transforming growth factor-beta in Alzheimer's disease. Neurology 45, 1561-1569.

Galvin, K.E., Ye, H., Erstad, D.J., Feddersen, R., and Wetmore, C. (2008). Gli1 Induces G2/M Arrest and Apoptosis in Hippocampal but Not Tumor-Derived Neural Stem Cells. STEM CELLS 26, 1027-1036.

Hendrickx, D.A.E., van Scheppingen, J., van der Poel, M., Bossers, K., Schuurman, K.G., van Eden, C.G., Hol, E.M., Hamann, J., and Huitinga, I. (2017). Gene Expression Profiling of Multiple Sclerosis Pathology Identifies Early Patterns of Demyelination Surrounding Chronic Active Lesions. Front Immunol 8, 1810. 
Hinks, G.L., and Franklin, R.J. (2000). Delayed changes in growth factor gene expression during slow remyelination in the CNS of aged rats. Mol Cell Neurosci 16, 542-556.

Hüttenrauch, M., Ogorek, I., Klafki, H., Otto, M., Stadelmann, C., Weggen, S., Wiltfang, J., and Wirths, O. (2018). Glycoprotein NMB: a novel Alzheimer's disease associated marker expressed in a subset of activated microglia. Acta Neuropathologica Communications 6,108 .

Ilzecka, J., Stelmasiak, Z., and Dobosz, B. (2002). Transforming growth factor-Beta 1 (tgf-Beta 1) in patients with amyotrophic lateral sclerosis. Cytokine 20, 239-243.

Issazadeh, S., Mustafa, M., Ljungdahl, A., Hojeberg, B., Dagerlind, A., Elde, R., and Olsson, T. (1995). Interferon gamma, interleukin 4 and transforming growth factor beta in experimental autoimmune encephalomyelitis in Lewis rats: dynamics of cellular mRNA expression in the central nervous system and lymphoid cells. J Neurosci Res 40, $579-590$.

Kanehisa, M. (2019). Toward understanding the origin and evolution of cellular organisms. Protein Science 28, 1947-1951.

Kanehisa, M., Furumichi, M., Sato, Y., Ishiguro-Watanabe, M., and Tanabe, M. (2021). KEGG: integrating viruses and cellular organisms. Nucleic Acids Research 49, D545D551.

Kanehisa, M., and Goto, S. (2000). KEGG: Kyoto Encyclopedia of Genes and Genomes. Nucleic Acids Research 28, 27-30.

Krupinski, J., Kumar, P., Kumar, S., and Kaluza, J. (1996). Increased expression of TGFbeta 1 in brain tissue after ischemic stroke in humans. Stroke 27, 852-857. 
Lindholm, D., Castren, E., Kiefer, R., Zafra, F., and Thoenen, H. (1992). Transforming growth factor-beta 1 in the rat brain: increase after injury and inhibition of astrocyte proliferation. J Cell Biol 117, 395-400.

Makwana, M., Jones, L.L., Cuthill, D., Heuer, H., Bohatschek, M., Hristova, M., Friedrichsen, S., Ormsby, I., Bueringer, D., Koppius, A., et al. (2007). Endogenous transforming growth factor beta 1 suppresses inflammation and promotes survival in adult CNS. The Journal of neuroscience : the official journal of the Society for Neuroscience 27, 11201-11213.

Massague, J. (1987). The TGF-beta family of growth and differentiation factors. Cell 49, 437-438.

Matsushima, G.K., and Morell, P. (2001). The neurotoxicant, cuprizone, as a model to study demyelination and remyelination in the central nervous system. Brain pathology (Zurich, Switzerland) 11, 107-116.

Mogi, M., Harada, M., Kondo, T., Narabayashi, H., Riederer, P., and Nagatsu, T. (1995). Transforming growth factor-beta 1 levels are elevated in the striatum and in ventricular cerebrospinal fluid in Parkinson's disease. Neurosci Lett 193, 129-132.

Moloney, E.B., Moskites, A., Ferrari, E.J., Isacson, O., and Hallett, P.J. (2018). The glycoprotein GPNMB is selectively elevated in the substantia nigra of Parkinson's disease patients and increases after lysosomal stress. Neurobiology of Disease 120, 1-11. Neal, M.L., Boyle, A.M., Budge, K.M., Safadi, F.F., and Richardson, J.R. (2018). The glycoprotein GPNMB attenuates astrocyte inflammatory responses through the CD44 receptor. Journal of neuroinflammation 15,73 . 
Nicaise, A.M., Wagstaff, L.J., Willis, C.M., Paisie, C., Chandok, H., Robson, P., Fossati, V., Williams, A., and Crocker, S.J. (2019). Cellular senescence in progenitor cells contributes to diminished remyelination potential in progressive multiple sclerosis.

Proceedings of the National Academy of Sciences 116, 9030-9039.

Owen, T.A., Smock, S.L., Prakash, S., Pinder, L., Brees, D., Krull, D., Castleberry, T.A., Clancy, Y.C., Marks, S.C., Jr., Safadi, F.F., et al. (2003). Identification and characterization of the genes encoding human and mouse osteoactivin. Crit Rev Eukaryot Gene Expr 13, 205-220.

Pasinetti, G.M., Hassler, M., Stone, D., and Finch, C.E. (1999). Glial gene expression during aging in rat striatum and in long-term responses to 6-OHDA lesions. Synapse 31, 278-284.

Plemel, J.R., Michaels, N.J., Weishaupt, N., Caprariello, A.V., Keough, M.B., Rogers, J.A., Yukseloglu, A., Lim, J., Patel, V.V., Rawji, K.S., et al. (2018). Mechanisms of lysophosphatidylcholine-induced demyelination: A primary lipid disrupting myelinopathy. Glia 66, 327-347.

R Core Team (2020). R: A Language and Environment for Statistical Computing (Vienna, Austria: R Foundation for Statistical Computing).

Radecki, D.Z., Messling, H.M., Haggerty-Skeans, J.R., Bhamidipati, S.K., Clawson, E.D., Overman, C.A., Thatcher, M.M., Salzer, J.L., and Samanta, J. (2020). Relative Levels of Gli1 and Gli2 Determine the Response of Ventral Neural Stem Cells to Demyelination. Stem Cell Reports 15, 1047-1055. 
Rimaniol, A.C., Lekieffre, D., Serrano, A., Masson, A., Benavides, J., and Zavala, F. (1995). Biphasic transforming growth factor-beta production flanking the proinflammatory cytokine response in cerebral trauma. Neuroreport 7, 133-136. Samanta, J., Burke, G.M., McGuire, T., Pisarek, A.J., Mukhopadhyay, A., Mishina, Y., and Kessler, J.A. (2007). BMPR1a Signaling Determines Numbers of Oligodendrocytes and Calbindin-Expressing Interneurons in the Cortex. The Journal of Neuroscience 27, 7397.

Samanta, J., Grund, E.M., Silva, H.M., Lafaille, J.J., Fishell, G., and Salzer, J.L. (2015). Inhibition of Gli1 mobilizes endogenous neural stem cells for remyelination. Nature 526, 448-452.

Samanta, J., Salzer, J.L. (2021). Transcriptomic analysis of loss of Gli1 in neural stem cells responding to demyelination in the mouse brain. NCBI GEO https://www.ncbi.nlm.nih.gov/geo/query/acc.cgi?acc=GSE162683.

Sanchez, M.A., and Armstrong, R.C. (2018). Postnatal Sonic hedgehog (Shh) responsive cells give rise to oligodendrocyte lineage cells during myelination and in adulthood contribute to remyelination. Exp Neurol 299, 122-136.

Sanchez, M.A., Sullivan, G.M., and Armstrong, R.C. (2018). Genetic detection of Sonic hedgehog (Shh) expression and cellular response in the progression of acute through chronic demyelination and remyelination. Neurobiol Dis 115, 145-156.

Satoh, J.I., Kino, Y., Yanaizu, M., Ishida, T., and Saito, Y. (2019). Microglia express GPNMB in the brains of Alzheimer's disease and Nasu-Hakola disease. Intractable Rare Dis Res $8,120-128$. 
Srinivasan, K., Friedman, B.A., Etxeberria, A., Huntley, M.A., van der Brug, M.P.,

Foreman, O., Paw, J.S., Modrusan, Z., Beach, T.G., Serrano, G.E., et al. (2020).

Alzheimer's Patient Microglia Exhibit Enhanced Aging and Unique Transcriptional

Activation. Cell reports 31, 107843.

Tanaka, H., Shimazawa, M., Kimura, M., Takata, M., Tsuruma, K., Yamada, M.,

Takahashi, H., Hozumi, I., Niwa, J., Iguchi, Y., et al. (2012). The potential of GPNMB as novel neuroprotective factor in amyotrophic lateral sclerosis. Sci Rep 2, 573.

Tarca, A.L., Draghici, S., Khatri, P., Hassan, S.S., Mittal, P., Kim, J.S., Kim, C.J.,

Kusanovic, J.P., and Romero, R. (2009). A novel signaling pathway impact analysis.

Bioinformatics (Oxford, England) 25, 75-82.

Utsunomiya, K., Owaki, K., Okumura, Y., Yano, M., Oto, T., Suzuki, E., Tamura, S., Abe, T., Kohno, S., Ohno, A., et al. (2012). An intracellular fragment of osteoactivin formed by ectodomain shedding translocated to the nucleoplasm and bound to RNA binding proteins. Bioscience, biotechnology, and biochemistry 76, 2225-2229.

Wachs, F.-P., Winner, B., Couillard-Despres, S., Schiller, T., Aigner, R., Winkler, J., Bogdahn, U., and Aigner, L. (2006). Transforming Growth Factor- $\beta 1$ Is a Negative Modulator of Adult Neurogenesis. Journal of Neuropathology \& Experimental Neurology $65,358-370$.

Wrana, J.L., Attisano, L., Wieser, R., Ventura, F., and Massague, J. (1994). Mechanism of activation of the TGF-beta receptor. Nature 370, 341-347.

Wyss-Coray, T., Borrow, P., Brooker, M.J., and Mucke, L. (1997). Astroglial overproduction of TGF-beta 1 enhances inflammatory central nervous system disease in transgenic mice. J Neuroimmunol 77, 45-50. 
Zhang, Y., Chen, K., Sloan, S.A., Bennett, M.L., Scholze, A.R., O'Keeffe, S., Phatnani, H.P., Guarnieri, P., Caneda, C., Ruderisch, N., et al. (2014). An RNA-Sequencing Transcriptome and Splicing Database of Glia, Neurons, and Vascular Cells of the Cerebral Cortex. The Journal of Neuroscience 34, 11929-11947.

Zhang, Y., Sloan, Steven A., Clarke, Laura E., Caneda, C., Plaza, Colton A., Blumenthal, Paul D., Vogel, H., Steinberg, Gary K., Edwards, Michael S.B., Li, G., et al. (2016).

Purification and Characterization of Progenitor and Mature Human Astrocytes Reveals Transcriptional and Functional Differences with Mouse. Neuron 89, 37-53. 
Radecki Page 24

\section{Figure Legends}

Figure 1 - Loss of Gpnmb increases differentiation of Gli1 vNSCs into oligodendrocytes following demyelination.

(A) RNAseq comparing Gli $1^{\text {NULL }}$ to Gli $1^{H E T}$ VNSCs, identified Gpnmb as the most significantly downregulated gene in Gli1 ${ }^{\text {NULL }}$ vNSCs following demyelination (Left). Gpnmb is significantly upregulated in Gli ${ }^{H E T}$ VNSCs on demyelination as compared to vNSCs from healthy mice (middle). Gpnmb is not induced upon demyelination in Gli1 ${ }^{\text {NULL }}$ vNSCs (right).

(B) Schematic of the experimental timeline for Cuprizone induced demyelination.

(C) Immunofluorescent images of the CC at 2 weeks of recovery from cuprizone diet showing NG2+ OPCs (green), CC1+ OLs (magenta) and LacZ+ Gpnmb expressing cells (yellow) in the CC. Scale $=30 \mu \mathrm{m}$

(D) Quantification shows a significant increase in CC1+ OLs in the Gpnmb ${ }^{\text {NULL }}$ CC compared to Gpnmb $^{W T}$ CC at 2 weeks of remyelination. $n=3$, data=mean \pm SEM, one-way ANOVA with post-hoc t-tests.

(E) Quantification for NG2+ OPCs in the CC showed no change at 2 wks of remyelination. n=4, 1-way ANOVA with post-hoc t-test.

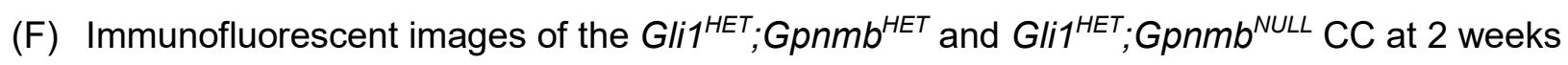
of remyelination showing RFP+ fate-mapped Gli1 vNSCs (magenta) co-expressing CC1 (green) and NG2 (green). Nuclei are counterstained with Hoechst. Scale $=25 \mu \mathrm{m}$.

(G) Quantification of percentage of RFP+ Gli1 fate-mapped vNSCs co-expressing NG2 and CC1, shows a significant increase in CC1+ OLs in Gli1 ${ }^{H E T}$;Gpnmb ${ }^{N U L L}$ CC compared to Gli $^{H E T}$;Gpnmb ${ }^{H E T} \mathrm{CC}$ at 2 weeks of remyelination. $\mathrm{n}=3$, unpaired t-tests within groups.

(H) Quantification of total number of RFP+ Gli1 fate-mapped cells in the Gli1 ${ }^{H E T}$;Gpnmb ${ }^{H E T}$ and Gli1 $^{\text {HET }}$;Gpnmb ${ }^{\text {NULL }}$ CC at 2 weeks of remyelination.

TGF $\beta 1-G p n m b$ signaling in vNSCs responding to demyelination 
DEM-demyelination, REM-remyelination, OPC-oligodendrocyte progenitor cells, OLoligodendrocytes, CC-corpus callosum

\section{Figure 2 - Expression of Gpnmb is limited to NSCs and glial cells in the forebrain}

(A) Immunofluorescent image showing co-localization of LacZ (magenta) and GFAP (green) in qNSCs (inset) in the SVZ (large dotted outline) of healthy Gpnmb ${ }^{H E T}$ mice. Scale $=25 \square \mathrm{m}$.

(B) Quantification of the percentage of LacZ+ cells co-expressing markers of qNSCs (GFAP), microglia (Iba1), oligodendrocytes (CC1), OPCs (PDGFRa) and neurons (NeuN), in the SVZ of healthy Gpnmb ${ }^{H E T}$ mice or at 1 week of recovery from cuprizone diet (REM). No colocalization with $\mathrm{NeuN}+$ neurons was detected. n=3, 2-way ANOVA with post-hoc t-tests within groups.

(C) Immunofluorescent images showing co-localization of LacZ (green) and RFP (magenta) in the healthy CC and after 1 week of remyelination in Gli ${ }^{H E T} ; G p n m b^{H E T}$ mice. Scale $=25 \mu \mathrm{m}$

(D) Quantification of (C) showing an increase in the proportion of RFP+ Gli1 vNSCs expressing LacZ at 1 week of recovery from cuprizone diet (REM) compared to healthy SVZ. $n=3$, unpaired t-test.

(E) Immunofluorescent image from Gpnmb ${ }^{H E T}$ mice showing PDGFRa+ OPCs and LacZ+ cells in the healthy forebrain and after 1 week of remyelination. Scale $=100 \mu \mathrm{m}$

(F) Immunofluorescent image from Gpnmb $b^{H E T}$ mice showing CC1+ OLs and LacZ+ cells in the healthy forebrain and after 1 week of remyelination. Scale $=100 \mu \mathrm{m}$

(G) Immunofluorescent images from Gpnmb ${ }^{H E T}$ mice showing GFAP+ astrocytes and LacZ+ cells in the healthy forebrain and after 1 week of remyelination. Scale $=100 \mu \mathrm{m}$

(H) Immunofluorescent images from Gpnmb ${ }^{H E T}$ mice showing Iba1+ microglia and LacZ+ cells in the healthy forebrain and after 1 week of remyelination. Scale $=100 \mu \mathrm{m}$

(I) Quantification of proportion of LacZ+ cells co-expressing cell-type specific markers in the healthy CC (solid bars) and at 1wk REM (thatched bars). There is a significant increase in 
the proportion of LacZ+ astrocytes and a decrease in microglia and OPCs. No colocalization with $\mathrm{NeuN}+$ neurons was detected. n=3, 2-way ANOVA with post-hoc t-tests within groups.

(J) Quantification of proportion of LacZ+ cells co-expressing cell-type specific markers in the healthy cortex (solid bars) and at 1wk REM (thatched bars). There is a significant increase in the proportion of LacZ+ astrocytes and a decrease in microglia. No co-localization with $\mathrm{NeuN}+$ neurons was detected. $\mathrm{n}=3,2$-way ANOVA with post-hoc t-tests within groups. ${ }^{*} p<.0001$.

Quiescent neural stem cell (qNSC), ventral neural stem cell (vNSC), Lateral ventricle (LV), corpus callosum (CC), cortex (Ctx), subventricular zone (SVZ), oligodendrocyte precursor cell (OPC), oligodendrocyte (OL), astrocyte (AS), microglia (MG)

\section{Figure 3 - TGF $\beta 1$ inhibits oligodendrogenesis by induction of Gpnmb expression.}

(A) KEGG pathway analysis of the RNAseq data showing activation of the TGF $\beta$ pathway in Gli $1^{H E T}$ vNSCs compared to Gli1 ${ }^{\text {NULL }}$ vNSCs upon demyelination.

(B) Schematic showing the CNS regions dissected for mRNA extraction (left). QRT-PCR for TGF $\beta 1$ expression in the CC, Ctx and SVZ tissue dissected from adult wildtype C57bl/6 mice fed with regular diet (Healthy) or cuprizone diet (DEM) for 3 weeks shows significant upregulation in the $\mathrm{CC}$ at $3 \mathrm{wks}$ demyelination (right). $n=3$, unpaired t-test within dissection regions.

(C) Experimental timeline for establishing in vitro culture of vNSCs.

(D) QRT-PCR for Gpnmb expression in vNSCs harvested from Gli1 ${ }^{\text {HET }}$ and Gli1 ${ }^{\text {NULL }}$ mice and treated with TGF $\beta 1$, BMP-4 and Shh in vitro, shows a significant increase in Gpnmb expression only in Gli1 ${ }^{H E T}$ vNSCs following TGF 1 treatment. n=3, 2-way ANOVA with post-hoc t-tests between all conditions. 
(E) Immunofluorescent images from Gli $1^{H E T}$ and Gli1 ${ }^{\text {NULL }}$ vNSCs following treatment with TGF 1 showing RFP+ cells derived from Gli1 vNSC and MBP+ OLs (insets). Scale $=25 \mu \mathrm{m}$

(F) Quantification of $(E)$ showing an increase in MBP+ OLs from Gli1 ${ }^{\text {NULL }}$ vNSCs upon TGF $\beta 1$ treatment, compared to Gli1 ${ }^{H E T}$ VNSCs. n=3, 1-way ANOVA with post-hoc t-tests between all groups.

(G) Immunofluorescent images from Gli $1^{\text {HET }}$ and Gli1 ${ }^{\text {NULL }}$ vNSCs following treatment with TGF $\beta 1$ showing NG2+ OPCs co-expressing RFP (insets). Scale $=25 \mu \mathrm{m}$

(H) Quantification of $(G)$ shows no difference in RFP+ OPCs generated from Gli1 ${ }^{\text {HET }}$ and Gli1 ${ }^{\text {NULL }}$ vNSCs with TGF 1 treatment. $n=3$, 1-way ANOVA with post-hoc t-tests between all groups.

(I) Immunofluorescent images from Gli $1^{\text {HET }}$ and Gli $1^{\text {NULL }}$ vNSCs following overexpression of Gpnmb using lentiviruses, showing GFP+ cells infected with the lentivirus (green), RFP+ cells derived from Gli1 vNSCs (magenta), and MBP+ OLs (yellow). Insets highlight cells showing co-localization of the markers- (1) GFP+RFP+MBP+ cell, (2) GFP+RFP+MBPcell, (3) GFP-RFP+MBP+ cell, (1') GFP-RFP-MBP+ cell, (2') GFP+RFP+MBP- cell, (3') GFP-RFP+MBP+ cell. Scale $=25 \mu \mathrm{m}$

(J) Quantification of the percentage of GFP+RFP+ OLs after 14 days of differentiation of vNSCs in vitro, showing significantly fewer OLs generated from both Gli1 ${ }^{\text {HET }}$ and Gli1 ${ }^{\text {NULL }}$ vNSCs upon Gpnmb overexpression (Gpnmb-OE) n=3, 1-way ANOVA with post-hoc t-tests between all groups.

Figure 4 - Conditional ablation of TGF $\beta R 2$ in vNSCs increases their recruitment and oligodendrogenesis in Gli1 ${ }^{\mathrm{NULL}}$ mice following demyelination

(A) QRT-PCR for the TGF $\beta 1$ receptor subunits, Tgfbr1 and Tgfbr2, with overexpression of Gpnmb (orange) or empty vector (white) in vNSCs harvested from adult wildtype C57bl/6 
mice, shows significant increase in the ligand binding Tgfbr2 subunit. $n=3$, one-way ANOVA with post-hoc t-tests within groups.

(B) QRT-PCR for expression of Gpnmb, TGF $\beta 1$ and TGF $\beta 1$ receptor subunits in the Gpnmb ${ }^{H E T}$ and Gpnmb ${ }^{\text {NULL }}$ SVZ, shows a significant decrease in Tgfbr2 expression. n=4, data=mean $\pm S E M$, unpaired $t$-test within gene groups.

(C) Schematic of the experimental timeline for lysophosphatidyl choline (LPC) induced demyelination.

(D) Immunofluorescent images showing co-localization of RFP (magenta) with CC1 (green) or

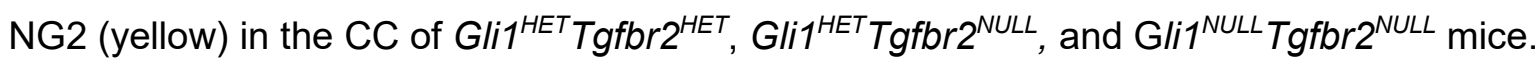
Scale $=30 \mu \mathrm{m}$

(E) Quantification of (D) shows increase in number of RFP+ cells derived from vNSCs in the corpus callosum (CC) of both Gli1 ${ }^{\text {HET }}$ and Gli $1^{\text {NULL }}$ mice upon loss of Tgfbr2. n=3, 2-way ANOVA with post-hoc t-tests between all groups.

(F) Quantification of percentage of RFP+ OPCs in the corpus callosum (CC) shows no change with loss of Tgfbr2 in Gli $1^{\text {HET }}$ and Gli $1^{\text {NULL }}$ mice following demyelination n=3, 1-way ANOVA with post-hoc t-tests.

(G) Quantification of percentage of RFP+ OLs in the corpus callosum (CC) shows an increase with loss of Tgfbr2 in Gli1 ${ }^{\text {NULL }}$ mice following demyelination n=3, 1-way ANOVA with posthoc t-tests.

\section{Extended Figure 1 - Gpnmb is expressed in multiple cell types and loss of Gpnmb does not alter extent of demyelination}

(A) Immunofluorescence for MBP expression in the corpus callosum (CC) after 5 weeks of cuprizone diet (DEM) showed no difference in the MBP+ area in Gpnmb ${ }^{W T}$ and Gpnmb $b^{\text {NULL }}$ mice. $n=3$, scale $=250 \mu m$, 1-way ANOVA with post-hoc t-test between groups. 
(B) Immunofluorescence of Gli11 ${ }^{H E T} ; G p n m b^{\text {NULL }}$ CC at 2 weeks of remyelination showing expression of Iba1 (yellow) and GFAP (green) along with fate-mapped Gli1 cells (RFP+). Quantification shows no change in the differentiation of RFP+ cells into astrocytes. Scale $=25 \mu \mathrm{m}, \mathrm{n}=3$, unpaired t-test.

(C) Immunofluorescence of the Gli ${ }^{\text {HET }}$;Gpnmb ${ }^{\text {NULL }}$ CC at 2 weeks of remyelination showing expression of LacZ in NG2+CC1 double positive cells indicating expression in premyelinating oligodendrocytes (left). Fate-mapped RFP+ cells in the CC co-expressing NG2, CC1, LacZ (inset). Scale $=30 \mu \mathrm{m}$.

(D) Quantification of proportion of RFP+ Gli1 fate-mapped cells co-expressing LacZ in the CC at 2 weeks of remyelination in Gli ${ }^{H E T} ; G p n m b^{H E T}$ and Gli ${ }^{H E T} ; G p n m b^{N U L L}$ mice. Unpaired ttests between groups.

(E) Analysis of published RNAseq and scRNAseq datasets identified Gpnmb expression in a variety of cells and tissues from mouse, human and rat, with lowest expression in mouse neurons.

\section{Extended Figure 2 - TGF $\beta 1$ does not alter differentiation of vNSC into astrocytes.}

(A) Immunofluorescenct images showing RFP+ vNSCs and GFAP+ astrocytes following treatment with vehicle or TGF $\beta 1$. Scale $=25 \mu \mathrm{m}$.

(B) Quantification of (A) showing a significant decrease in proportion of RFP+ cells coexpressing GFAP in Gli1 ${ }^{\text {NULL }}$ vNSCs treated with TGF $\beta 1$ compared to Gli ${ }^{\text {HET }}$ vehicle controls. $n=3$, data=mean \pm SEM, 1-way ANOVA with post-hoc t-tests between groups. 


\section{Extended Data 3 - Gpnmb overexpression does not alter differentiation of vNSC into} OPCs or astrocytes.

(A) QRT-PCR for Gpnmb mRNA expression shows significant increase in vNSCs overexpressing Gpnmb (Gpnmb-OE) compared to empty vector. n=4, data=mean \pm SEM, unpaired t-test.

(B) Quantification of the percentage of GFP+ lentiviral infected vNSCs shows similar rates of infection in Gli1 ${ }^{\text {HET }}$ and Gli $1^{\text {NULL }}$ vNSCs. n=3, data=mean \pm SEM, 1-way ANOVA with post-hoc t-tests.

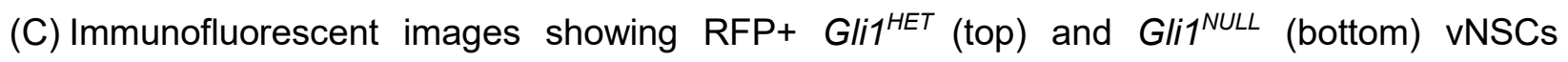
(magenta), infected with the GFP or Gpnmb-overexpression (Gpnmb-OE) lentivirus (green), co-expressing OPC markers, NG2 or PDGFRa (yellow). Scale $=25 \mu \mathrm{m}$.

(D) Quantification of (C) showing no difference in percentage of OPCs generated from the RFP+ Gli ${ }^{1 H E T}$ and Gli $i^{1 N U L L}$ vNSCs, infected with the GFP or Gpnmb-overexpression (Gpnmb-OE) lentivirus. $n=3,1$-way ANOVAs within groups, followed by post-hoc t-tests for comparisons within the lentiviral treatment conditions and genotypes.

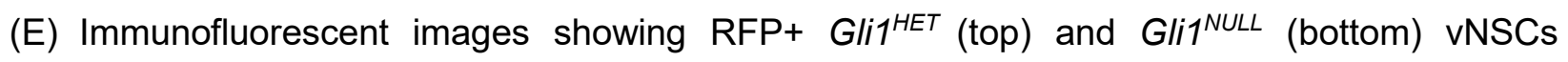
(magenta), infected with the GFP or Gpnmb-overexpression (Gpnmb-OE) lentivirus (green), co-expressing the astrocyte marker, GFAP (yellow). Scale $=25 \mu \mathrm{m}$.

(F) Quantification of (E) showing no difference in percentage of astrocytes generated from the RFP+ Gli ${ }^{H E T}$ and Gli1 ${ }^{\text {NULL }}$ vNSCs, infected with the GFP or Gpnmb-overexpression (Gpnmb-OE). n=3, 1-way ANOVAs within cell groups, followed by post-hoc t-tests for comparisons within the lentiviral treatment conditions and genotypes.

\section{Extended Data 4 - Loss of TGF $\beta R 2$ reduces pSmad3 signaling in Gli1 vNSCs and does not alter the extent of demyelination.}

TGF $\beta 1-G p n m b$ signaling in vNSCs responding to demyelination 
(A) Immunofluorescent images showing GFAP+ quiescent neural stem cells (yellow), RFP+ vNSCs (magenta) and pSmad3 (green). Scale $=25 \mu \mathrm{m}$.

(B) Immunofluorescent images showing MBP+ area, 2 days after stereotaxic injection of saline or lysophosphatidyl choline (LPC) into the CC of Gli1 ${ }^{H E T} T G F \beta R 2^{N U L L}$ mice (top), Gli $^{\text {HET TGF }}{ }^{\text {HR }} 2^{\text {HET }}$ (middle) and Gli1 ${ }^{\text {HET }}$ TGF $\beta R 2^{\text {NULL }}$ (bottom) mice. Scale $=250 \mu \mathrm{m}$

\section{Supplemental Table 1 -Genotypes of mice.}

The table lists the genotypes of all the mice, their stock numbers, and their abbreviated names.

TGF $\beta 1-G p n m b$ signaling in vNSCs responding to demyelination 


\section{MATERIALS AND METHODS}

\section{Lead Contact}

Further information and requests for resources and reagents should be directed and will be fulfilled by the lead contact Jayshree Samanta (Jayshree.samanta@wisc.edu).

\section{Materials availability}

The pLV-Gpnmb overexpression plasmid is available and will be provided by the lead contact, Jayshree Samanta ( Jayshree.samanta@wisc.edu) upon request.

\section{Data and code availability}

The Gli1 NSC RNAseq is available online (GSE162683), and all other GSE datasets are currently publicly available. All analysis code is available online, and no custom code was created for or from this project.

Fate mapping and cuprizone demyelination: All animals were used and maintained according to protocols approved by the University of Wisconsin IACUC. These mouse lines were obtained from Jackson labs or KOMP repository: Gli1 ${ }^{\text {CreERT2 }}$ (Jax\# 007913), ROSA26Sor ${ }^{\text {tm9(CAG-tdTomato)Hze (Jax\# 007909), Tgfbr2 }}{ }^{\text {tm1Karl }}$ (Jax\# 012603), Gpnmb ${ }^{\text {tm1.1(KOMP)Vlcg }}$ (Mutant Mouse Resource \& Research Center (MMRRC) \#047926-UCD). The genotypes for the mouse lines are as follows: Gpnmb ${ }^{\mathrm{HET}}=\mathrm{Gpnmb}^{\mathrm{tm} 1.1(\mathrm{KOMP}) \mathrm{Vlcg} /+}, \mathrm{Gpnmb}^{\mathrm{NULL}}=$ $\mathrm{Gpnmb}^{\mathrm{tm} 1.1(\mathrm{KOMP}) \mathrm{Vlcg} / \mathrm{tm} 1.1}, \mathrm{Ai9}=\mathrm{ROSA} 26 \mathrm{Sor}^{\mathrm{tm} 9 / \mathrm{tm} 9} ; \mathrm{Gli}^{\mathrm{HET}}=\mathrm{Gli}^{\mathrm{CreERT2/+}} ; \mathrm{Ai9}, \mathrm{Gli}^{\mathrm{NULL}}=$

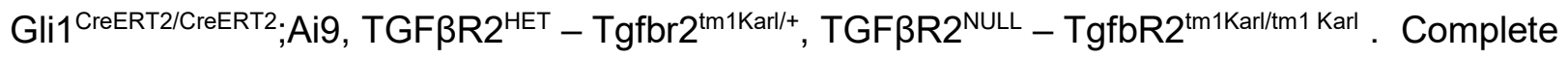
genotypes and abbreviated names are summarized in Table S1. All the mice were maintained on $\mathrm{C} 57 \mathrm{BI} / 6$ background. 12-16 week old mice were administered $5 \mathrm{mg}$ tamoxifen (Sigma) in corn oil on alternate days for a total of four intraperitoneal injections. No labelling was seen in the absence of tamoxifen administration. A week later, demyelination was induced by feeding $0.2 \%$ cuprizone in the chow for 5 weeks following which the diet was returned to normal chow for recovery from demyelination. 
Stereotaxic Surgery: 1 week prior to surgery, mice received 4 i.p. injections of $5 \mathrm{mg}$ tamoxifen every other day. 1\% lysophosphatidylcholine (LPC) (L4129, Millipore Sigma) was dissolved in sterile saline to a final concentration of $10 \mathrm{mg} / \mathrm{mL}$. Mice were injected with $0.3 \mathrm{mg} / \mathrm{kg}$ Buprenorphine 15-30min prior to induction of anesthesia, and then once every 12 hours for up to 48 hours post-surgery depending on recovery. $4-5 \%$ isoflurane with $1 \mathrm{~L} / \mathrm{min}$ oxygen supplementation was used to induce anesthesia until steady, rhythmic $\sim 60 \mathrm{breaths} / \mathrm{min}$ breathing was observed, following which mice were transferred to a nose cone with a bite bar on Stoelting digital stereotaxic frame with built in heated base maintained at $38^{\circ} \mathrm{C}$ for the duration of surgery. Once immobilized in the frame, the dorsal fur of the skull was cleaned $3 x$ with $70 \%$ ethanol, then a posterior-anterior incision was made to expose the skull. A $2 \mathrm{~mm}$ burr hole was drilled at the following coordinates: $0.5 \mathrm{~mm}$ AP $\times 1.0 \mathrm{~mm} \mathrm{ML} \times-1.9 \mathrm{~mm}$ DV (measured from the surface of the skull). A 10uL 33G Hamilton NEUROS syringe (\#53497) was lowered and $1 \mathrm{uL}$ of $1 \% \mathrm{LPC}$ or $1 \mathrm{uL}$ of sterile saline was injected over $5 \mathrm{~min}$. The needle was slowly retracted, the wound sealed with Vetbond (\#1469SB, 3M) and mice were moved to a $37^{\circ} \mathrm{C}$ chamber to recover until they became mobile. Mice were monitored daily for recovery, and sacrificed 1 week following surgery.

Primary NSC culture: The brains were harvested from mice after euthanasia and placed in an acrylic mouse brain mold (BS-A-5000C, Braintree Scientific) and 1mm slices were acquired from the caudal aspect of the olfactory bulbs to the dorsal hippocampus. Brain slices were moved to dissection media composed of DMEM/F-12 (\#11320032, Gibco) with 1x Antibiotic-Antimycotic (\#15240112 - Gibco) and the SVZ tissue lining the ventral and lateral aspects of the lateral ventricles was microdissected from 3 consecutive slices. The dissected SVZ tissue was minced and collected by centrifugation at $400 \mathrm{~g}$ for $5 \mathrm{~min}$. The tissue was then digested with $0.05 \%$ TrypsinEDTA (\#25300062, Gibco) and incubated at $37^{\circ} \mathrm{C}$ for $5 \mathrm{~min}$ with agitation every $60-90$ secs to form a cell suspension. After neutralizing the Trypsin-EDTA with $1 \mathrm{mg} / \mathrm{mL}$ Soybean Trypsin Inhibitor (\#17075029, Gibco), the cells were needle triturated and resuspended in Mouse NSC Proliferation TGF $\beta 1-G p n m b$ signaling in vNSCs responding to demyelination 
Media (1x Anti-Anti, 10ng/mL bFGF (\#78003, Stem Cell Tech, Vancouver), 20ng/mL mEGF (\#78016, Stem Cell Tech), 0.0002\% Heparin Sulfate (\#H7640, Sigma), 1x Mouse Proliferation Supplement (\#5701) in NeuroCult Media (\#5702 Stem Cell Tech). The dissociated cells were then grown as floating neurospheres in proliferation media at $37^{\circ} \mathrm{C}$ and $5 \% \mathrm{CO}_{2}$. Primary NSCs were supplemented with fresh bFGF and mEGF at day 3 and day 5 after plating. Neurospheres were passaged at day 7 to generate secondary neurospheres. Secondary neurospheres were dissociated into single cells and plated on Matrigel (\#356234 Corning, concentration tested by WiCell) coated coverslips in differentiation media (1x Anti-Anti, 1ng/mL bFGF, 2ng/mL mEGF, 0.0002\% Heparin, 1x Differentiation Supplement in Neurocult Media (\#5704 Stem Cell Tech Kit). Cells were maintained in Differentiation Media for 7 days with half media changes every other day, then harvested for RNA or fixed in ice-cold methanol for $15 \mathrm{~min}$ for immunolabelling.

Lentivirus generation: HEK293T cells were cultured to 40\% confluency in T-175 flasks in RPMI1640 (R8758, Corning) with 10\% fetal bovine serum (SH30068.03, Hyclone) and 1x Penicillin/Streptomycin (15-140-122, Gibco). Cells were transfected with either pLV-eGFP plasmid (\#36083, Addgene) or pLV-Gpnmb-eGFP (custom synthesis from Synbiotech) in combination with pMD2.G (\#12259, Addgene) and psPAX2 (\#12260, Addgene) in equivalent molar ratios. Viafect reagent (E4981, Promega) was utilized at a 6:1 ratio for transfection as per the manufacturer's instructions. Briefly, Viafect was added to pre-warmed serum-free media, followed by addition of all plasmids with gentle mixing and incubation at room temperature for 20min. The plasmid/Viafect mixture was added to cells and allowed to incubate for 12 hours, followed by full media change. The supernatant was collected at 24 and 48 hours following the media change and pooled together before filtering through a pre-cooled $0.22 \square \mathrm{m}$ cell filter, then pelleting via ultracentrifugation for 3hours at 75,000xg in a swinging bucket rotor. The supernatant was then aspirated, leaving a residual $50-100 \square$ I which was removed by inverting tubes onto absorbent cloth. Lentivirus pellet was then resuspended in $100 \square$ I LentiGuard reagent (Cellomics) 
and stored at $-80^{\circ} \mathrm{C}$ until use. The average titer for all lentiviruses was $\sim 2 \times 10^{6} \mathrm{TU} / \mathrm{mL}$ and was used at an $\mathrm{MOI}=0.13$.

In vivo immunostaining: Mice were perfused transcardially with $4 \%$ PFA, the brains were harvested and then allowed to equilibrate for 12 hours in $30 \%$ sucrose. Tissue was then embedded and frozen in OCT media, and $20 \mu \mathrm{m}$ coronal cryosections were obtained and processed for immunofluorescence. Slides were thawed and washed 3 times in 1xPBS for 5 min each, followed by blocking in a solution of $1 \times \mathrm{PBS} / 0.1 \% \mathrm{BSA} / 0.3 \%$ TritonX-100 with $10 \%$ normalized goat serum. Then combinations of the following antibodies: rat anti-PDGFRa (1:200, BD Biosciences), rat anti-RFP (1:1000, Chroma Tek); chicken anti-GFP (1:100, Invitrogen), mouse anti-CC1 (1:400, MilliporeSigma), anti-GFAP (1:400, Sigma), anti-MBP (1:500, Millipore) and anti-LacZ (1:2,000, Sigma) and rabbit anti-NG2 (1:200, MilliporeSigma), anti-pSmad3 (1:100 Abcam) were applied by diluting antibodies in the $1 \mathrm{xPBS} / 0.1 \% \mathrm{BSA} / 0.3 \%$ TritonX-100 solution and incubating in a humid chamber overnight at $4^{\circ} \mathrm{C}$. Slides were then washed in $1 \times$ PBS 3 times for $5 \mathrm{~min}$ each, and goat anti-species secondary antibodies conjugated with Alexafluor 488,568 , or $647(1: 1,000$, Invitrogen) were added to the $1 \times P B S / 0.1 \% B S A / 0.3 \%$ TritonX-100 solution and nuclei were simultaneously counterstained with Hoechst 33342 (1:5,000, Invitrogen). Secondary antibodies were added to sections for 1 hour, followed by 3 washes with 1 xPBS for 5 min each, and 1 wash with filtered water for $5 \mathrm{~min}$. All liquid was then removed, without allowing the slides to dry followed by coverslipping the sections with Fluoromount-G before sealing them.

Immunofluorescence image acquisition and analysis: Total RFP+ cells were counted using a Nikon E600 Eclipse Epifluorescent microscope with wavelength specific bandpass cubes at 20x magnification. Counting was performed by examiners blinded to the genotype and experimental condition. Epifluorescent images were obtained as Z-stacks of $5 \mu \mathrm{m}$ optical sections using a Keyence BZ-X700 microscope at 20x magnification. These images were used for single channel of total labelled cell populations in the CC, SVZ (defined as a $<20 \square \mathrm{m}$ region around the lateral ventricles), and cortex (sensorimotor cortex directly dorsal to the $\mathrm{CC}$ region from cingulum to TGF $\beta 1-G p n m b$ signaling in vNSCs responding to demyelination 
cingulum). For total CC area, 4x images were obtain on Keyence for all brain slices analyzed (89 slices/mouse) and manually segmented for total area. For imaging of co-localized markers, $1 \square \mathrm{m}$ optical sections were obtained on a laser scanning Leica TCS SP8 confocal microscope with LasX software. Regions of interest were stitched, merged and flattened for co-localization, and Zstack projections were used to confirm positive cells. Results were collated in Microsoft Excel before analysis in GraphPad Prism. The investigators were blinded to allocation during image analysis and outcome assessment.

In vitro immunostaining: After 14 days of differentiation, NSC cultures were fixed by washing cells once with HBSS, then fixed with $500 \mathrm{uL}$ of cold methanol for $15 \mathrm{~min}$. Cells were grown on coverslips, which were removed from wells using fine tweezers and placed on Parafilm. This allows for reduced liquid usage as the hydrophobic Parafilm prevents liquid from leaving the coverslips. Cells were then washed 3 times with 1xPBS for 5 min each, then blocked in a solution of $1 \times$ PBS with $0.1 \%$ bovine serum albumin (BSA) with $0.3 \%$ Trinton $X-100$ and $10 \%$ normalized goat serum for 1 hour at room temperature. Following blocking, primary antibodies, chicken antiGFP (1:100), rat anti-RFP (1:2000), rabbit anti-RFP $(1: 1000)$, rabbit anti-NG2 (1:100), rat antiPDGFR $\square(1: 100)$, mouse anti-MBP (1:100) and mouse anti-GFAP (1:1000), were added to the 1xPBS/0.1\%BSA/0.3\%TritonX-100 solution with combinations of primary antibodies depending on isotype and presence of GFP or RFP in cells. NSCs were incubated overnight at $4^{\circ} \mathrm{C}$ in a dark humid chamber. Coverslips were then washed 3 times with 1 XPBS for $5 \mathrm{~min}$ each, then secondary antibodies (1:1000) were added with Hoechst reagent (1:5000) for 1 hour at room temperature. Coverslips were then washed 3 times in 1 XPBS followed by 1 wash with distilled water, then inverted onto a drop of Fluoromount-G on clean slides and allowed to settle overnight. Images from in vitro studies were acquired using Keyence BZ-X700 epifluorescent microscope from 10 fields per coverslip, across a total of 3 coverslips per marker per experimental group. Each experiment was repeated 3 times and the cell counts were averaged together. Total RFP+ (fatemapped) cells were counted using Keyence software batch analysis counting by first counting all TGF $\beta 1-G p n m b$ signaling in vNSCs responding to demyelination 
nuclei in the image via a threshold analysis, then confirming which nuclei were overlaid with RFP for the fate-mapped cells. This analysis was confirmed to be in $>95 \%$ concordance with manual counts. Differentiated NG2+ (OPCs), MBP+ (OLs), and GFAP+ (Astrocytes) cells were counted manually using ImageJ.

In vivo qRT-PCR: For in vivo qRT-PCR, adult (90-120 day old) mice were sacrificed by cervical dislocation and the brain was removed and placed into the coronal $1 \mathrm{~mm}$ mouse brain mold. $1 \mathrm{~mm}$ sections were obtained beginning caudal to the olfactory bulbs through the dorsal hippocampus. Brain slices from $0.5 \mathrm{~mm}-1.0 \mathrm{~mm}$ relative to Bregma were identified and placed into dissection media (ice cold DMEM/F-12 with 1x Anti-Anti (\#15240-062, Gibco) under a dissecting microscope. The cortex spanning $-3.0 \mathrm{~mm}$ to $3.0 \mathrm{~mm}$ relative to midline was removed by making vertical cuts to the corpus callosum (CC), then dissecting along the dorsal aspect of the $\mathrm{CC}$. $\mathrm{CC}$ was then removed by inserting scissors into the dorsal aspects of the lateral ventricles and cutting along the dorsal border of the lateral ventricles. Finally, SVZ was removed by dissecting around the lateral ventricles, using fiber bundles in the striatum as a guide for the limit of the SVZ tissue. Immediately following dissection, tissue was frozen in pre-cold tubes on dry ice. Total mRNA was extracted using Trizol-chloroform following the manufacturer's instructions, and concentration and purity was determined using a NanoDrop Lite (ND-LITE-PR, Thermo Fisher). mRNA was reversetranscribed to complementary DNA using iScript cDNA Synthesis Kit (BioRad). ITaq Universal SYBR Green Supermix (BioRad) was used to perform qPCRs in a Biorad CFX Connect thermal cycler.

In vitro qRT-PCR: Differentiated NSCs plated in 24-well plates were washed with 1x PBS, then lifted using $0.05 \%$ Trypsin-EDTA for $5 \mathrm{~min}$ at $37^{\circ} \mathrm{C}$. An equal volume of $1 \mathrm{mg} / \mathrm{mL}$ Trypsin Inhibitor in DMEM/F-12 was added to neutralize Trypsin-EDTA, and cells were physically lifted using a cell scraper; 3 wells were pooled to generate a single technical replicate. Total mRNA was isolated using Monarch Total RNA Miniprep Kit and concentration and quality was confirmed using NanoDrop Lite. cDNA was synthesized and qPCR was run as described above.

TGF $\beta 1-G p n m b$ signaling in vNSCs responding to demyelination 
Bioinformatics Analysis: For the overview of Gpnmb expression across samples, NCBI Gene Expression Omnibus was manually searched for Affymetrix array, bulk RNAseq or scRNAseq data sets related to mouse, rat, or human oligodendroglia, all glia, or total brain cell types. Transformed data sets were queried for reporting on Gpnmb expression, and if present, the rank expression value within each experimental replicate was recorded and averaged for reporting.

For bulk RNAseq analysis of Gli ${ }^{H E T}$ and Gli $1^{\text {NULL }}$ SVZ, sample preparation, RNA collection and sequencing were performed as described in (Samanta, 2021) and the data were deposited to NCBI-GEO (GSE162683). Volcano plots were generated using the package EnhancedVolcano v1.8.0 (Blighe et al., 2020) under R v4.0.3 (R Core Team, 2020). Genes with adjusted p-values less than or equal to 0.05 and absolute values of log2 fold change greater or equal to 0.6 were considered as significant differentially expressed genes (DEGs).

The KEGG pathway analysis was done by using Advaita Bio's iPathwayGuide (version 1902) (Ahsan and Drăghici, 2017; Donato et al., 2013; Draghici et al., 2007; Tarca et al., 2009). All measured gene expressions from the RNA sequencing data were imported into the iPathwayGuide platform, and the same thresholds of DEGs as the volcano plots were used. The iPathwayGuide utilizes the Impact Analysis method, which considers the over-representation of DEGs in the pathway and the perturbation of pathway based on the pathway topology from the KEGG database, to determine the significance of each pathway (Draghici et al., 2007; Kanehisa, 2019; Kanehisa et al., 2021; Kanehisa and Goto, 2000). The calculated p-values were then corrected for multiple tests via the Benjamini-Hochberg method (Benjamini and Hochberg, 1995) to control the false discovery rate (FDR).

Statistical Analysis: All results were validated by at least three independent technical and biological experiments. At least 5 sections per mouse were analyzed and data from 3-5 mice, with a mix of male and female sexes, were combined to determine the average, standard deviation, and SEM. The quantitative data were expressed as mean \pm SEM. Statistical analysis 
bioRxiv preprint doi: https://doi.org/10.1101/2021.08.13.456269; this version posted August 14, 2021. The copyright holder for this preprint (which was not certified by peer review) is the author/funder. All rights reserved. No reuse allowed without permission.

Radecki Page 39

was performed using Student's t test, 1-way ANOVA, or 2-way ANOVA with Tukey's post hoc ttest. Differences were considered statistically significant at $p<0.05$.

TGF $\beta 1-G p n m b$ signaling in vNSCs responding to demyelination 
Figure 1

A Gli1 NULL DEM vs.

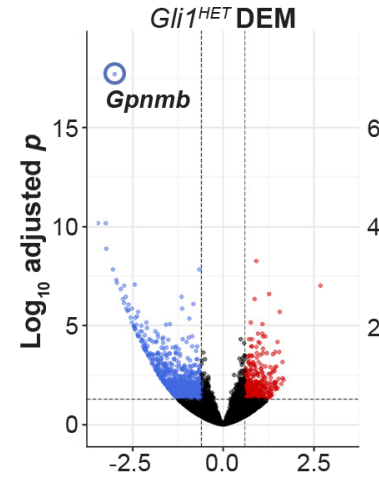

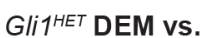
Gli ${ }^{\text {HET }}$ Healthy

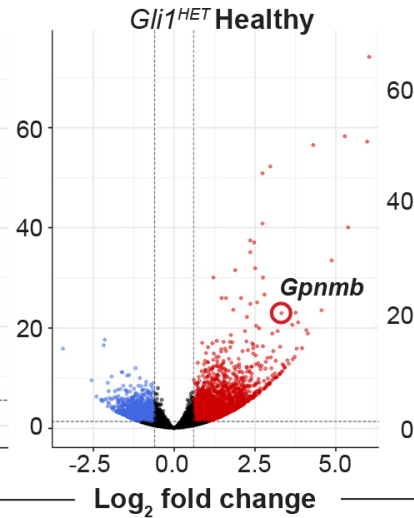

C
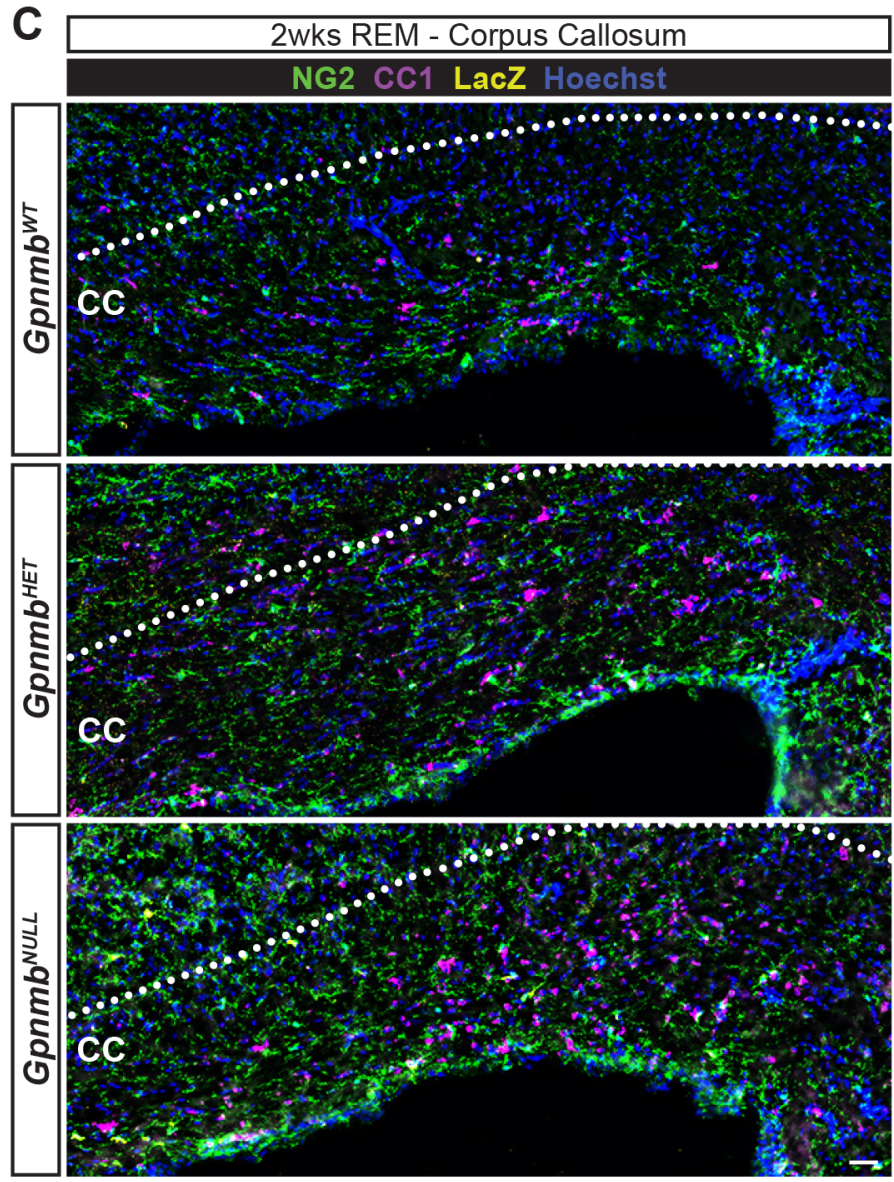

$\mathbf{F}$
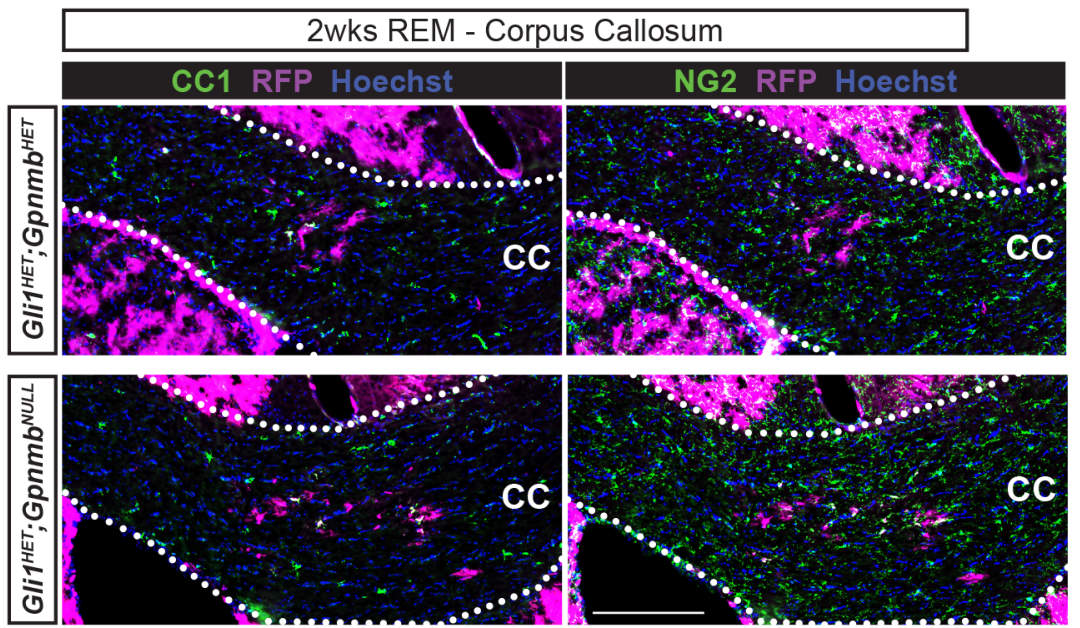

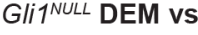
Gli1 ${ }^{\text {NULL }}$ Healthy

B

Experimental Timeline

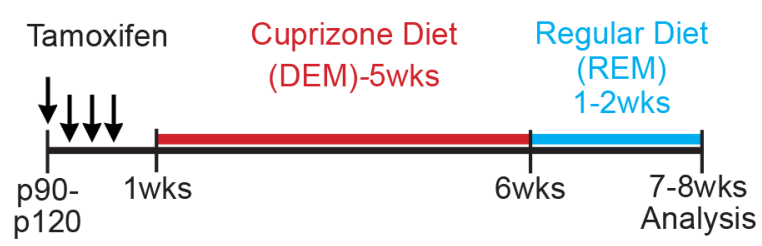

E

CC
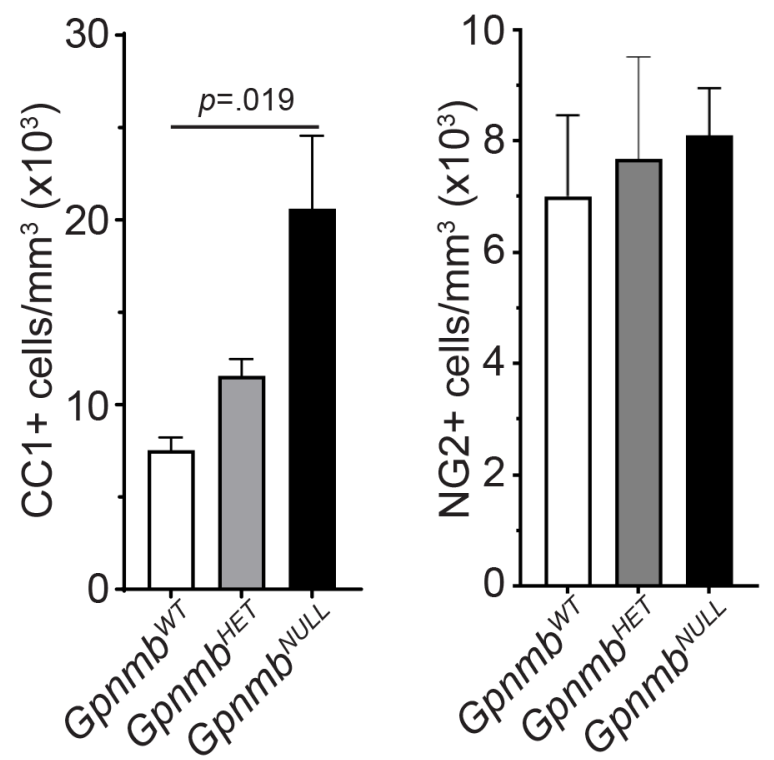

G Fate-mapped H Fate-mapped OL Lineage

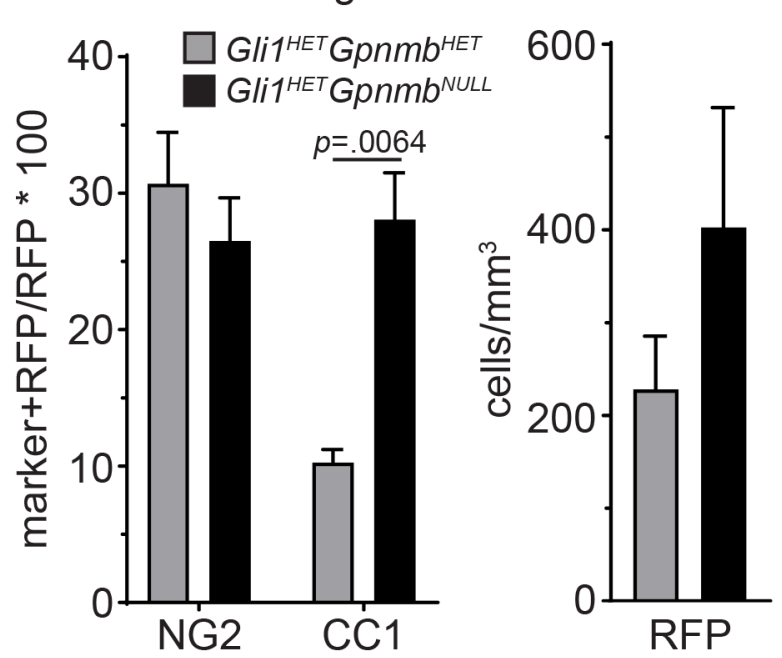


Figure 2
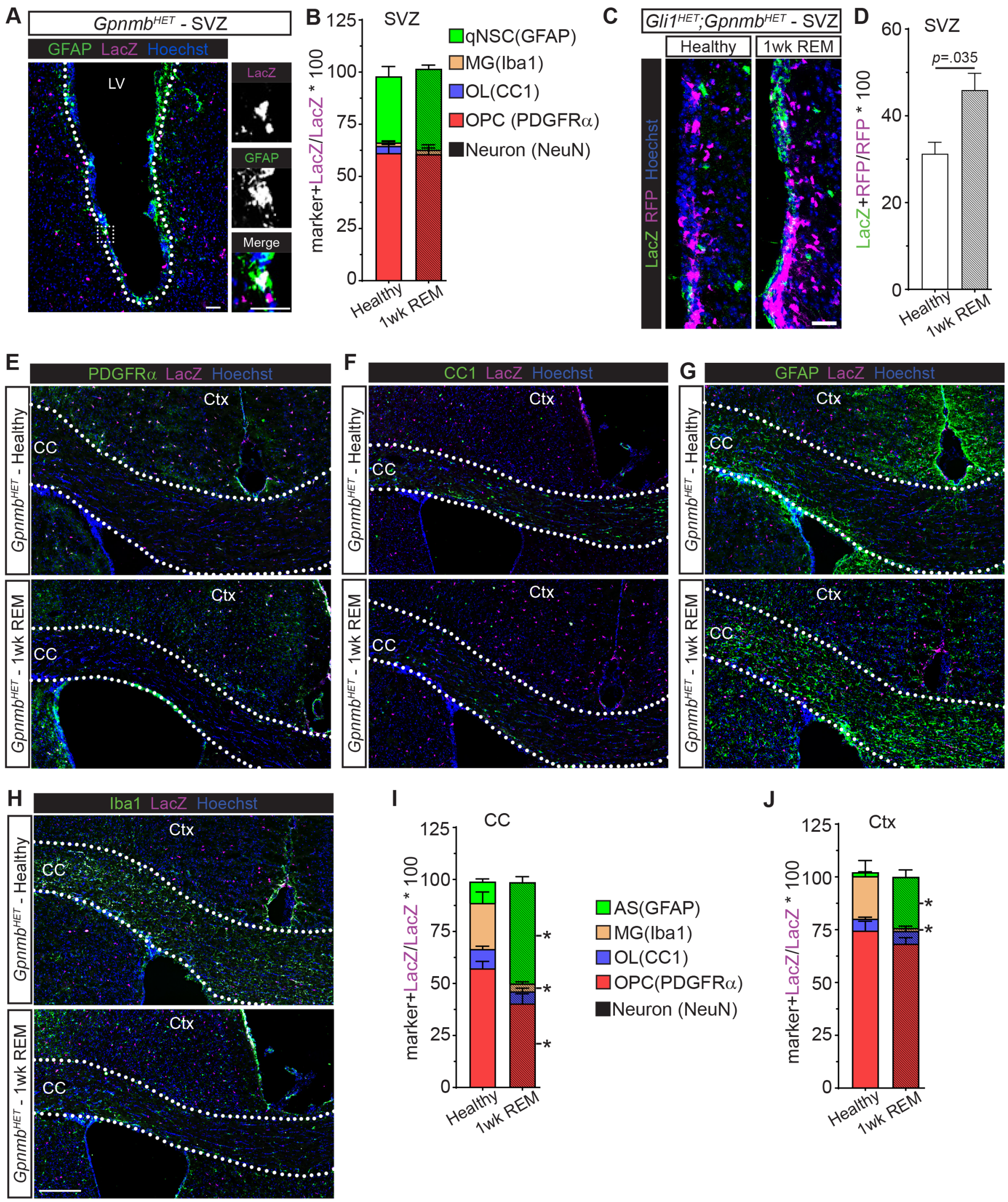


\section{Figure 3}

A

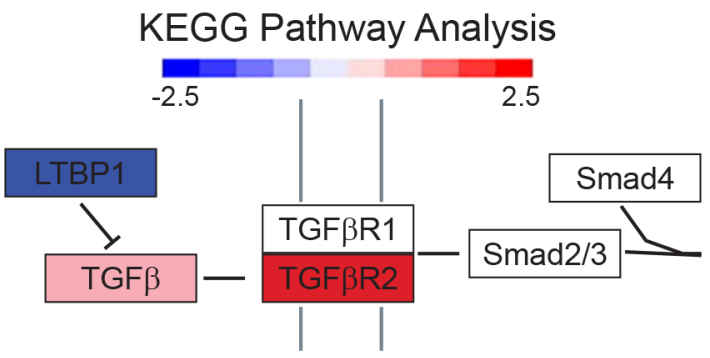

B

Cortex $=\mathrm{Ctx}$

Corpus Callosum $=\mathrm{CC}$

Subventricular zone $=$ SVZ

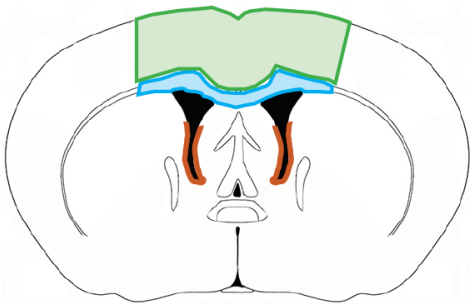

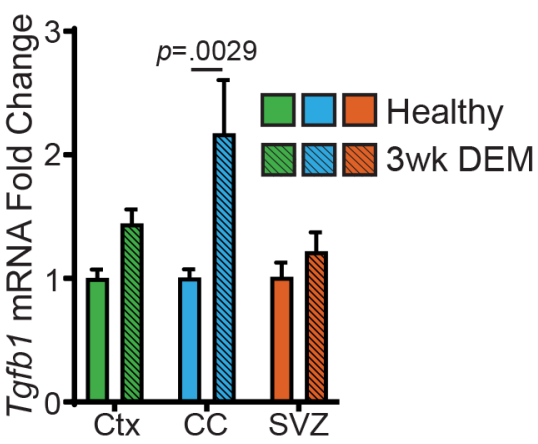

D

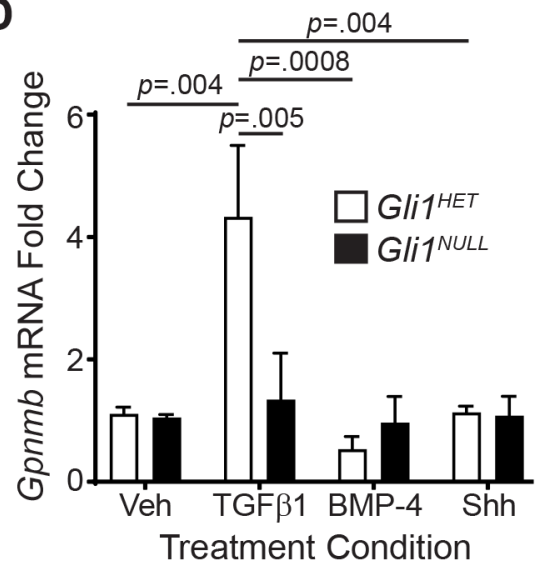

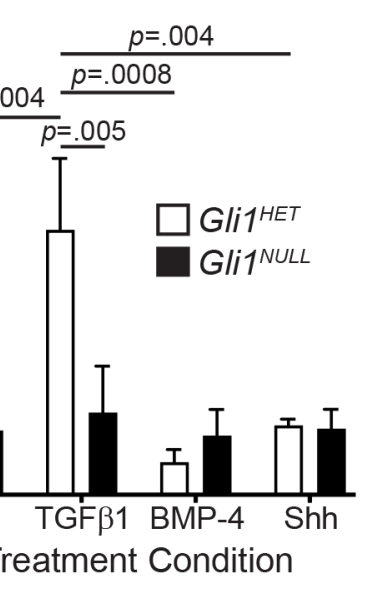

E

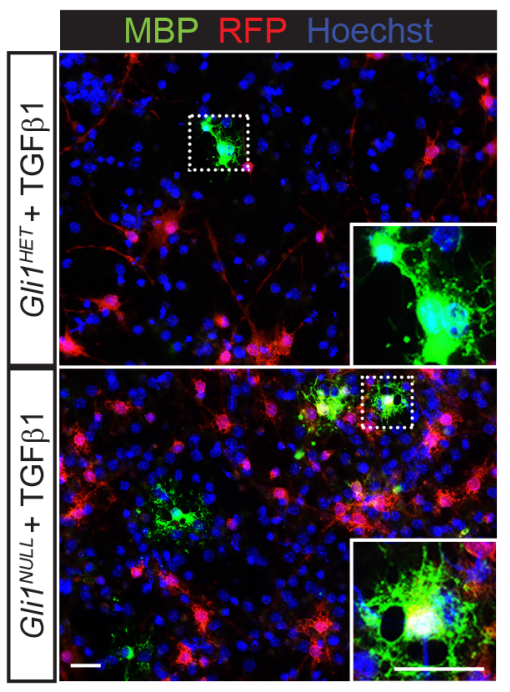

F Veh TGF $\beta 1$ Gli1HET
Gli1NULL $\square \square$

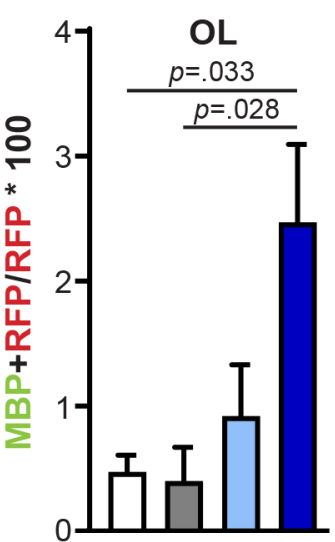

G

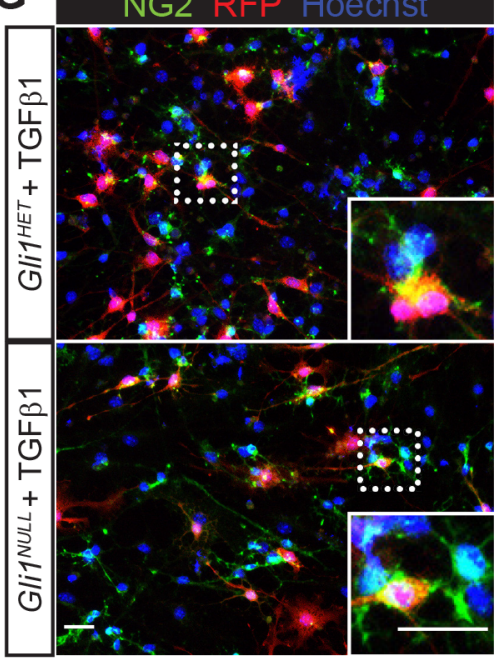

H Veh TGF $\beta 1$ Gli1HET $\square \square$
Gli1NULL $\square$

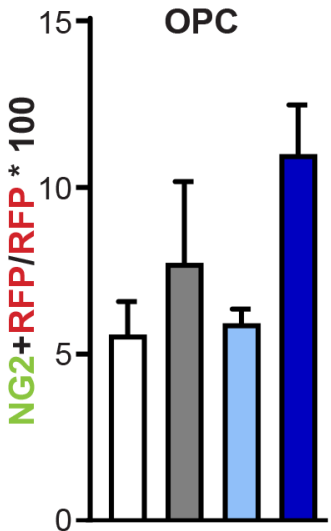

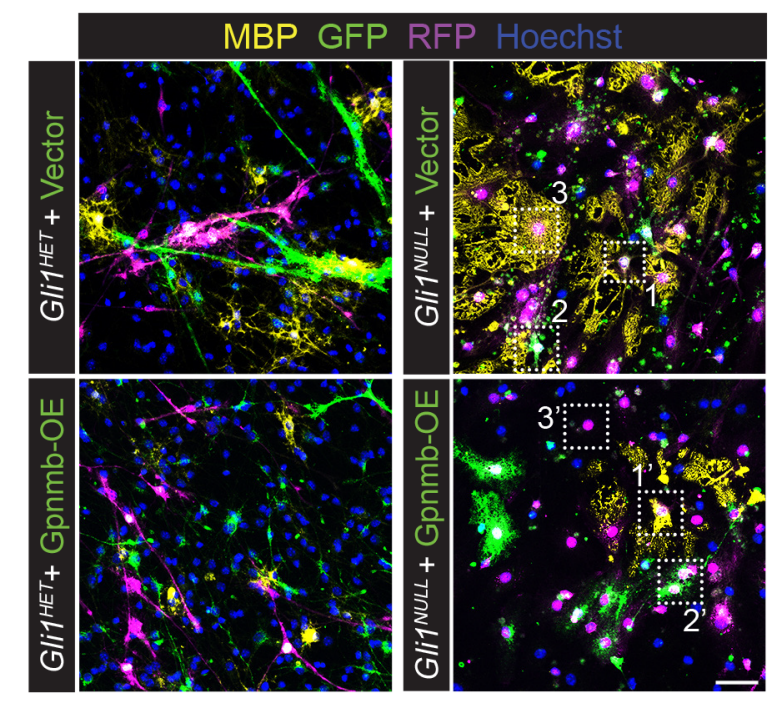

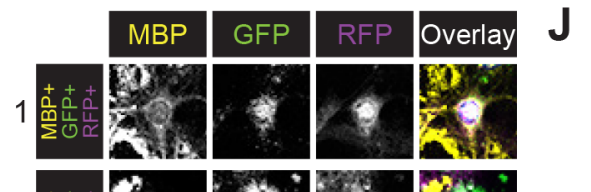

Vector Gpnmb-OE

$\square$ Gli1HET D

Gli1 NULL
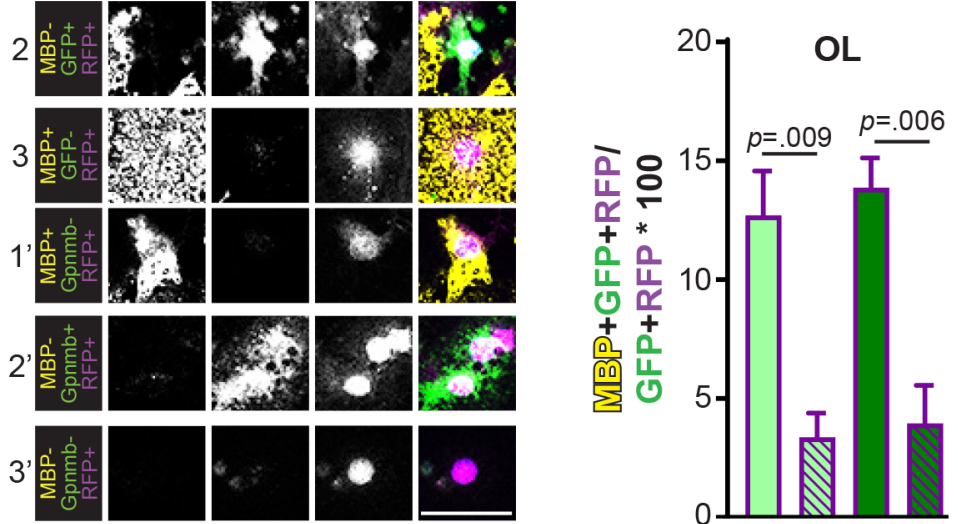


\section{Figure 4}

A Lentiviral Transduction

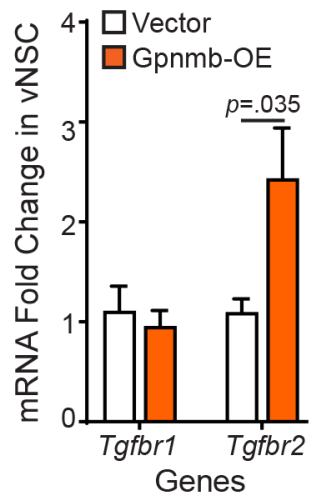

D
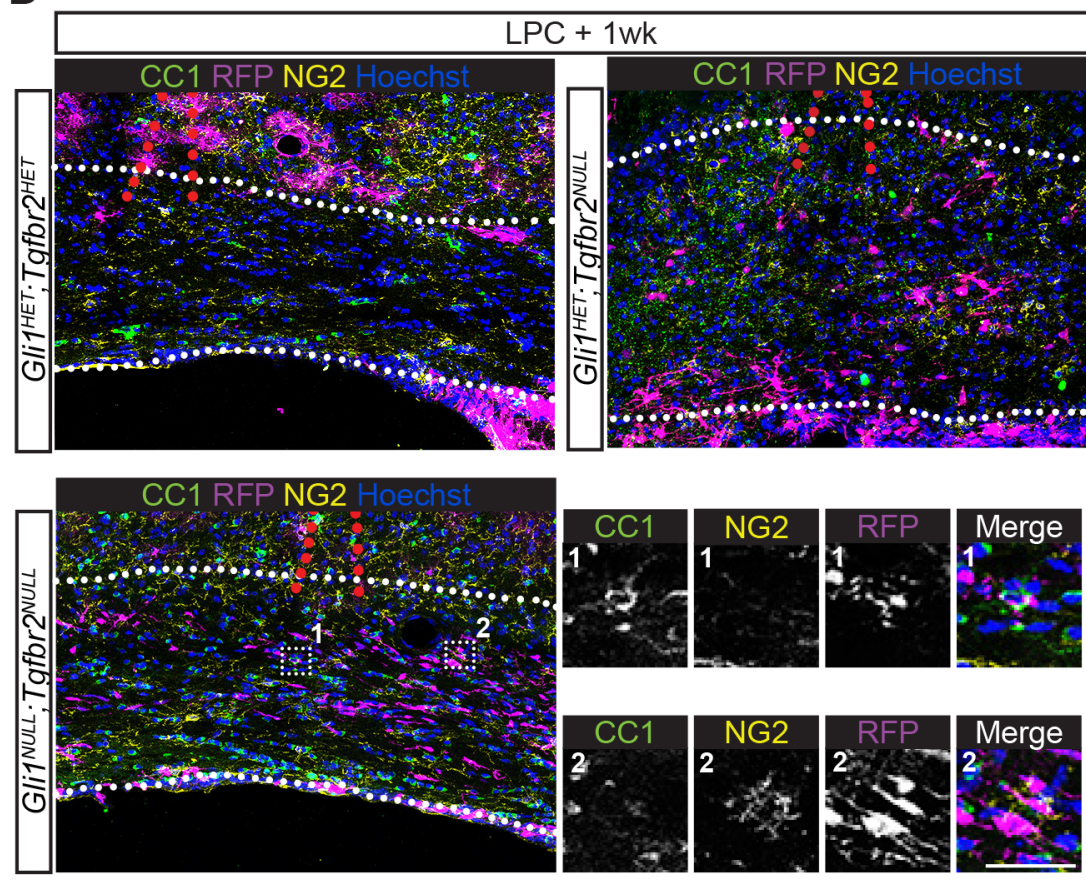

F
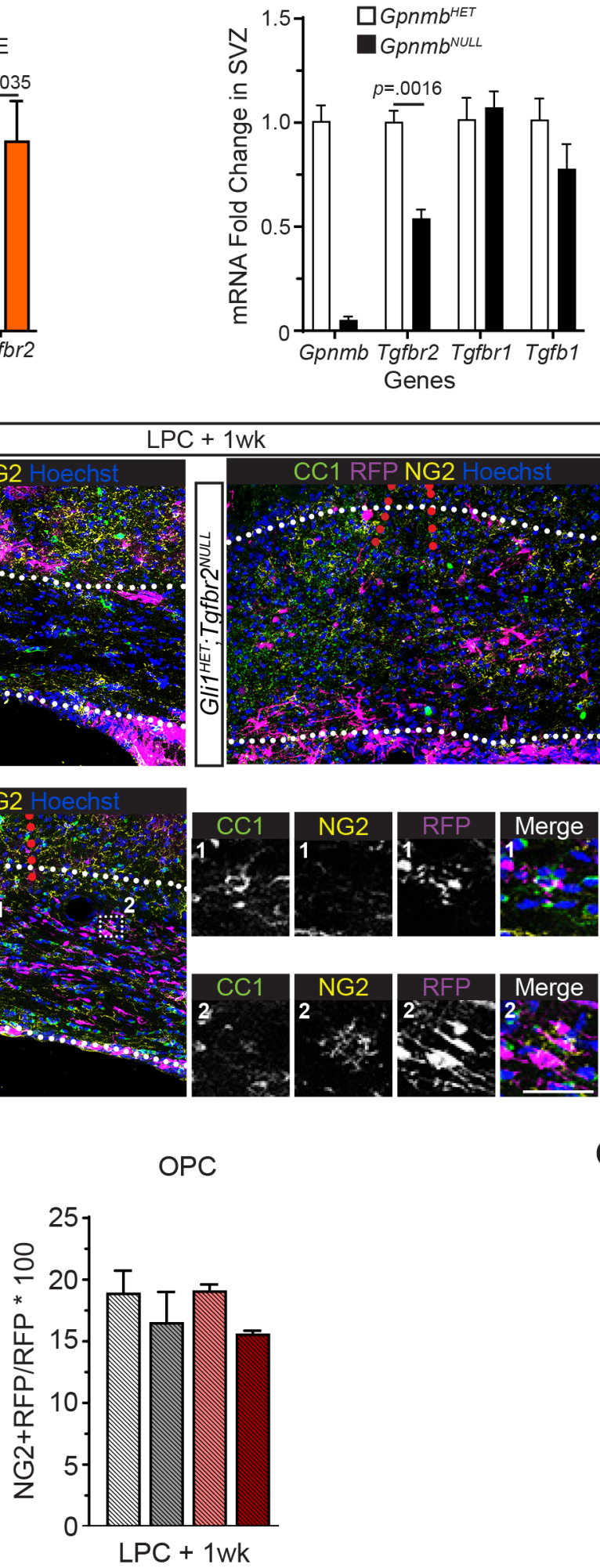

C

E

Veh LPC

$\square \quad$ Gli1 $^{\text {HET }}$;gfbr2 ${ }^{\text {HET }}$

$\square$ Gli1HET;Tgfbr2 ${ }^{\text {NULL }}$

$\square$ Gli1 GULL;Tgfbr2 $^{\text {HET }}$

$\square$ Gli1 ${ }^{\text {NULL }} ;$ Tgfbr2 $2^{\text {NULL }}$

Total RFP cells in CC

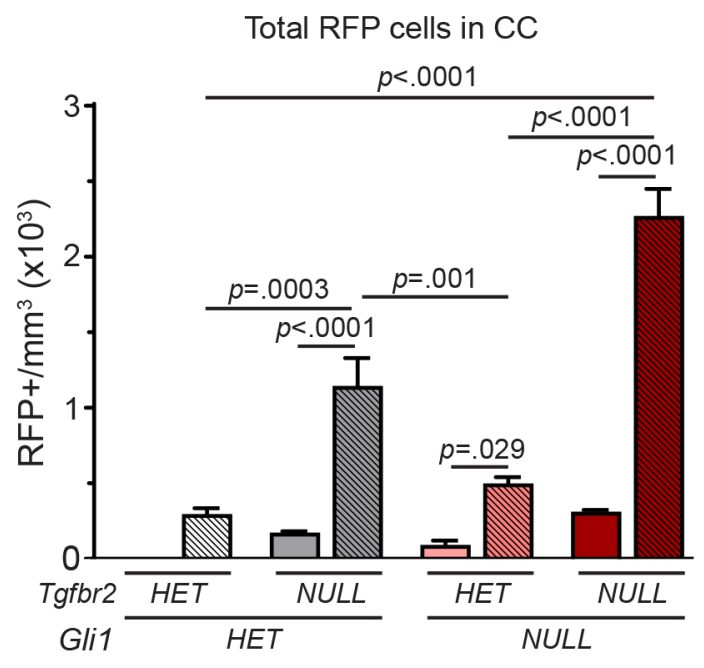

G

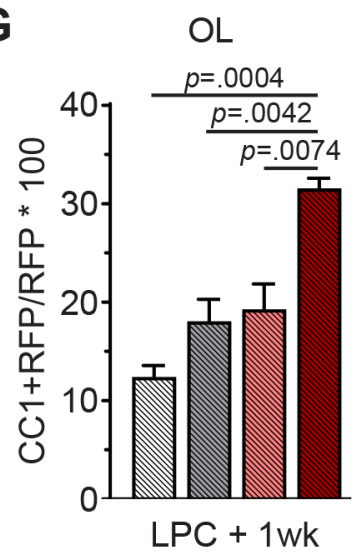

fib
Analysis

2wk
Experimental Timeline

Stereotaxic Surgery Vehicle

$1 w k$

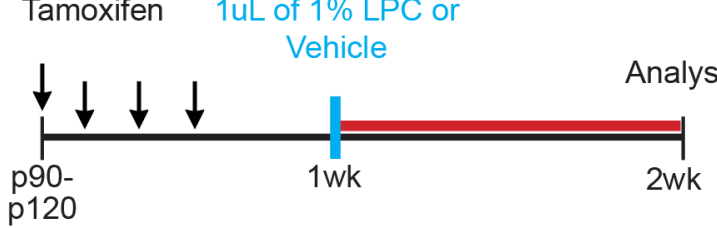




\section{Figure S1}

A
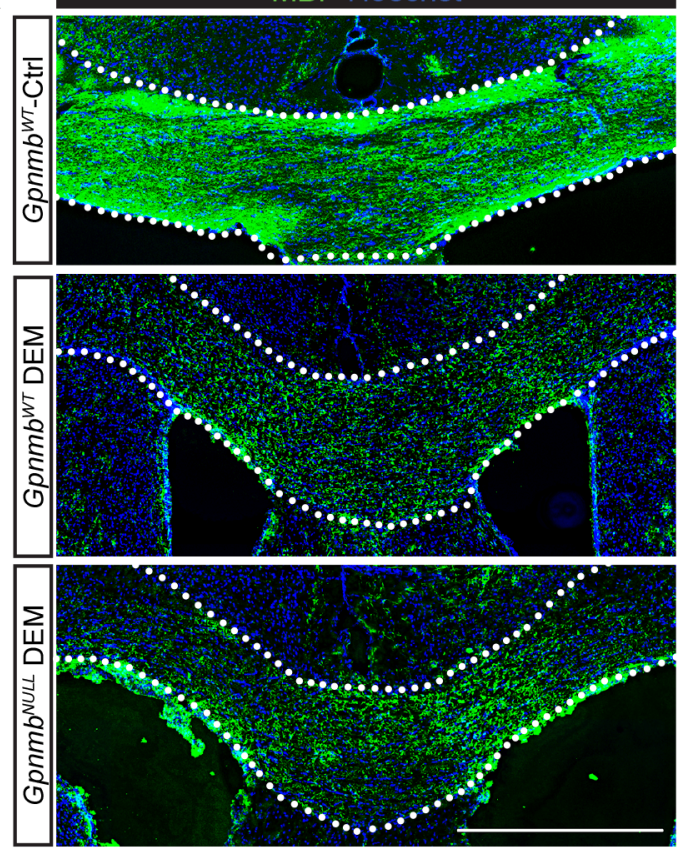

B

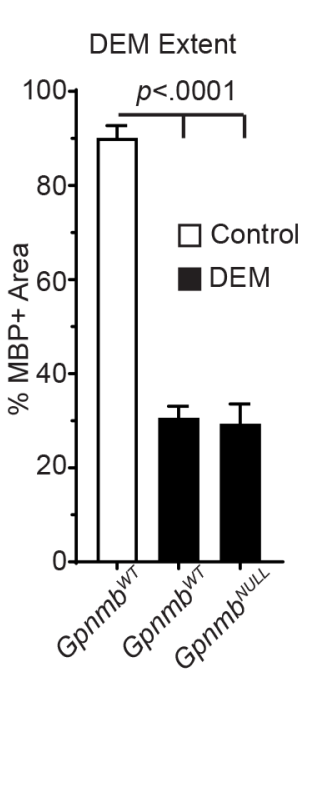

2wk REM - Gli1 ${ }^{\text {HET; }}$ Gpnmb ${ }^{\text {NULL }}$
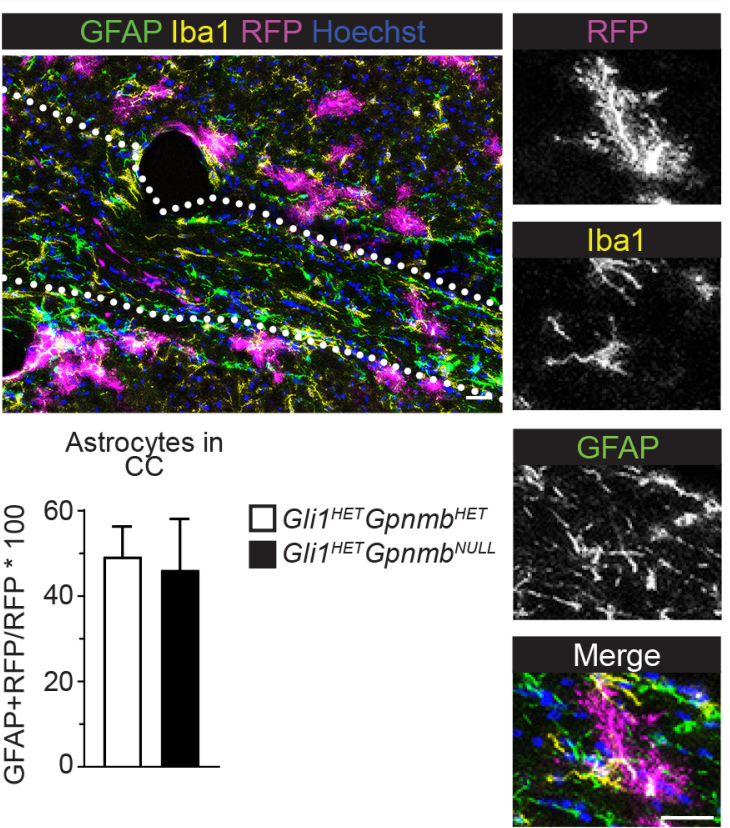

c

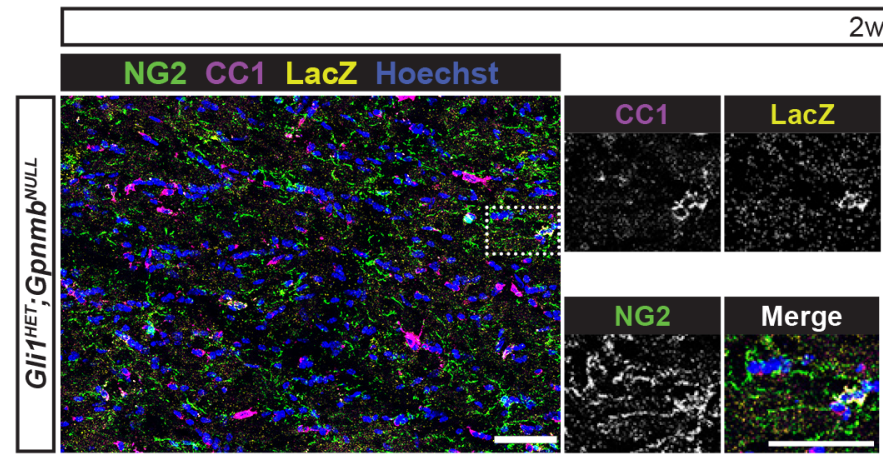

WW REM - CC

CC

D

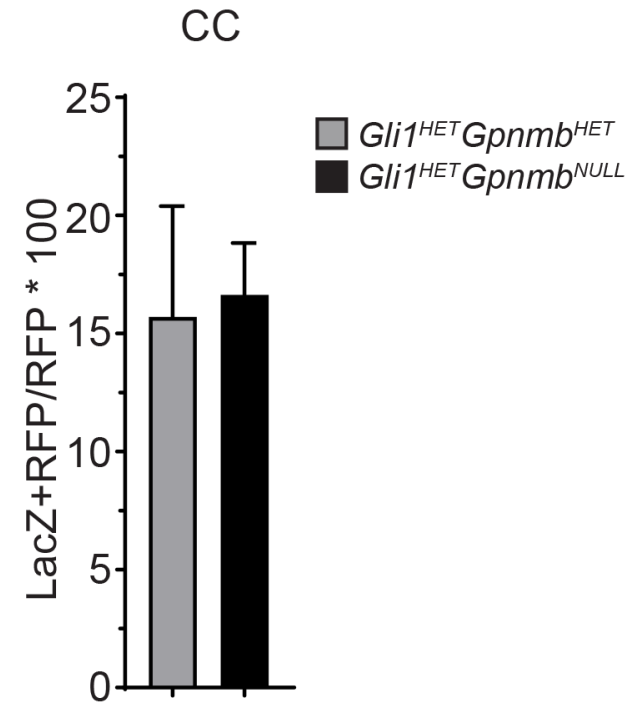

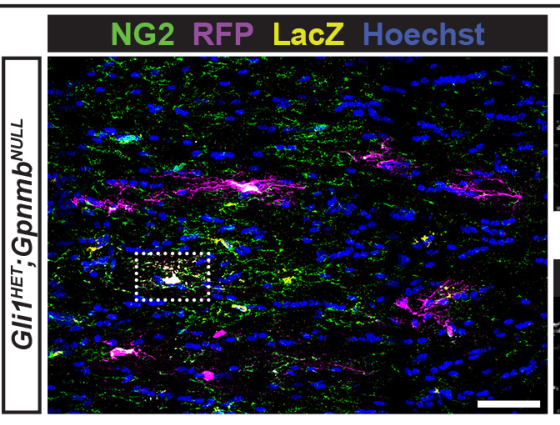

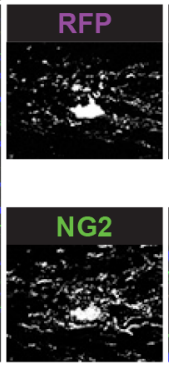

LacZ

E

Gpnmb Expression

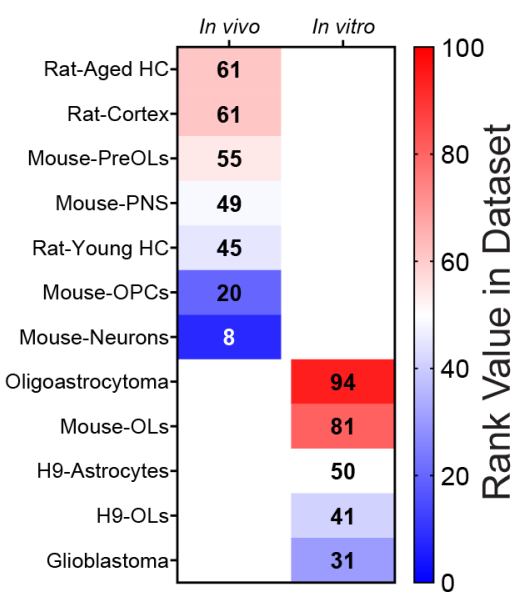


Figure S2

A
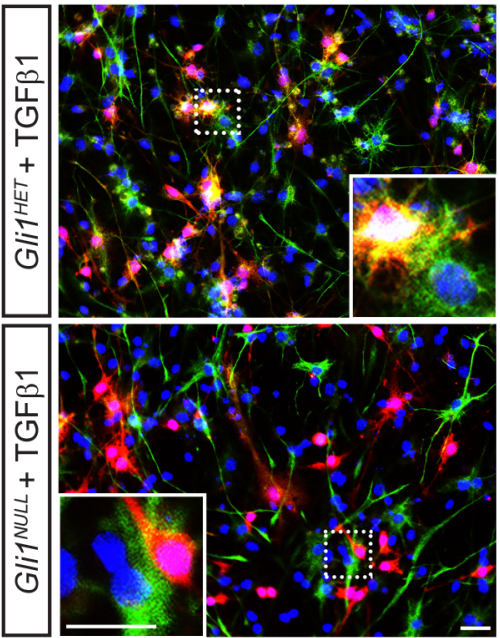

B

Veh TGF $\beta 1$

Gli1HET

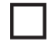

Gli1 ${ }^{\text {NULL }}$
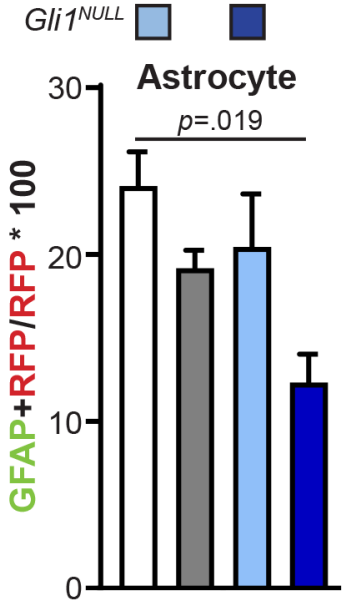


\section{Figure S3}

A vNSC + Lentivirus

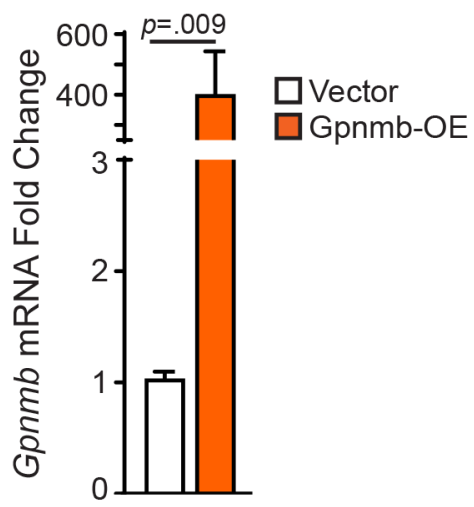

B vNSC + Lentivirus

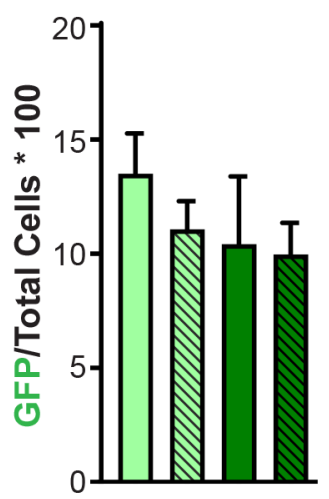

Vector Gpnmb-OE

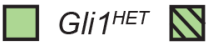

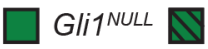

C
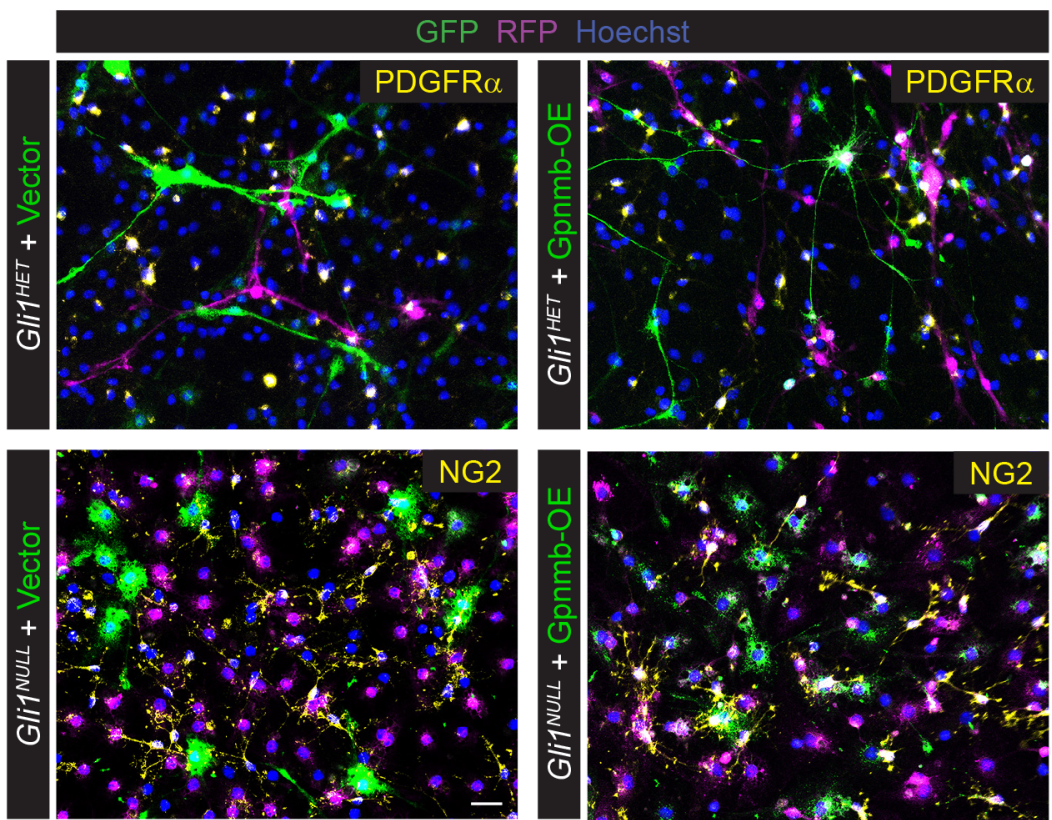

E
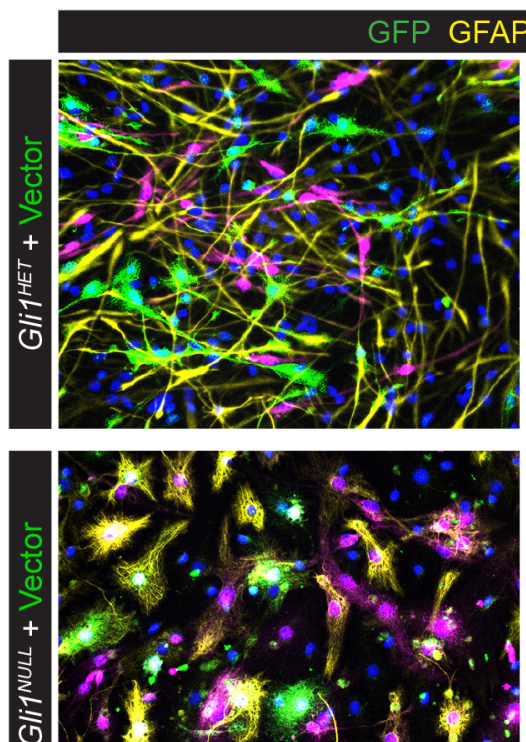
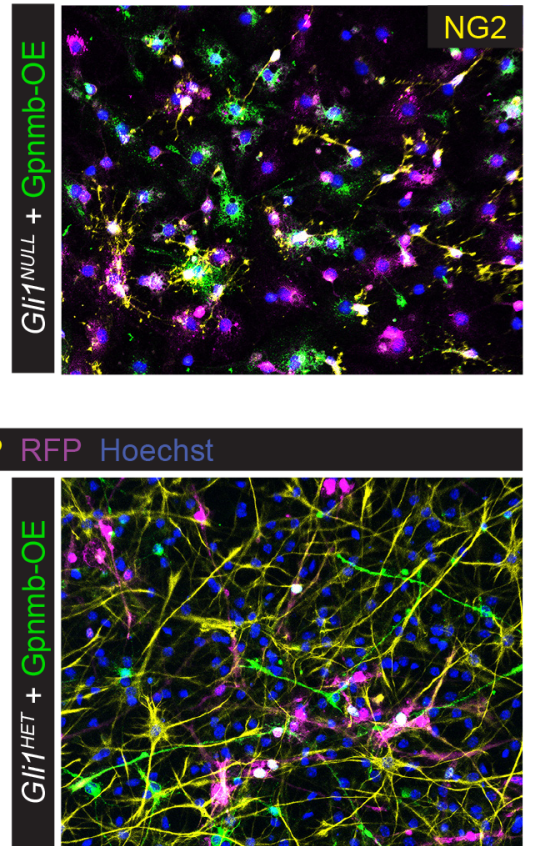

D

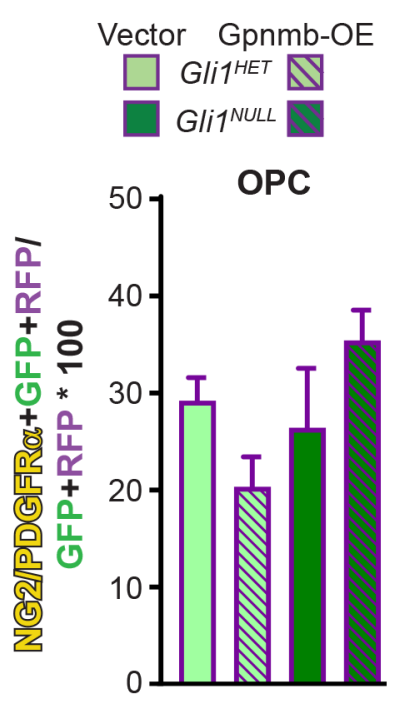

$\mathbf{F}$

Vector Gpnmb-OE $\square$ Gli1HET Gli1NULL

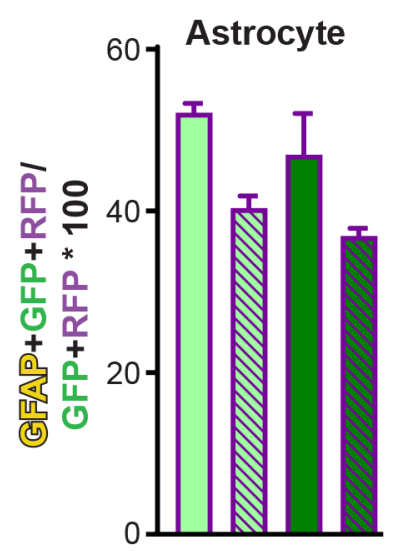


Figure S4

A

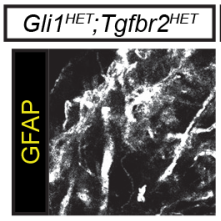

Gli1 $^{\text {HET; Tgfbr2 }}{ }^{\text {NULL }}$
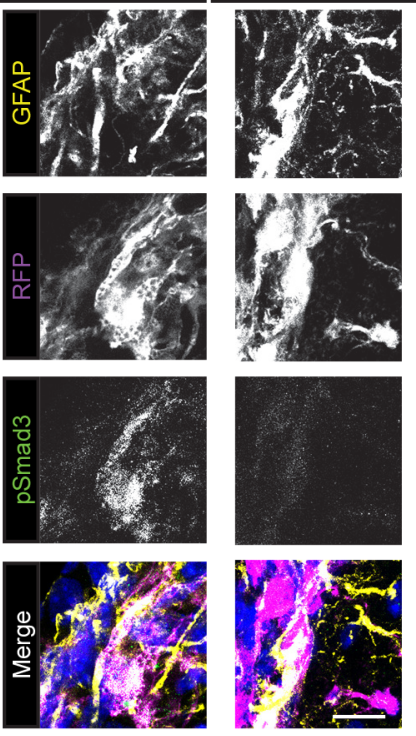

B
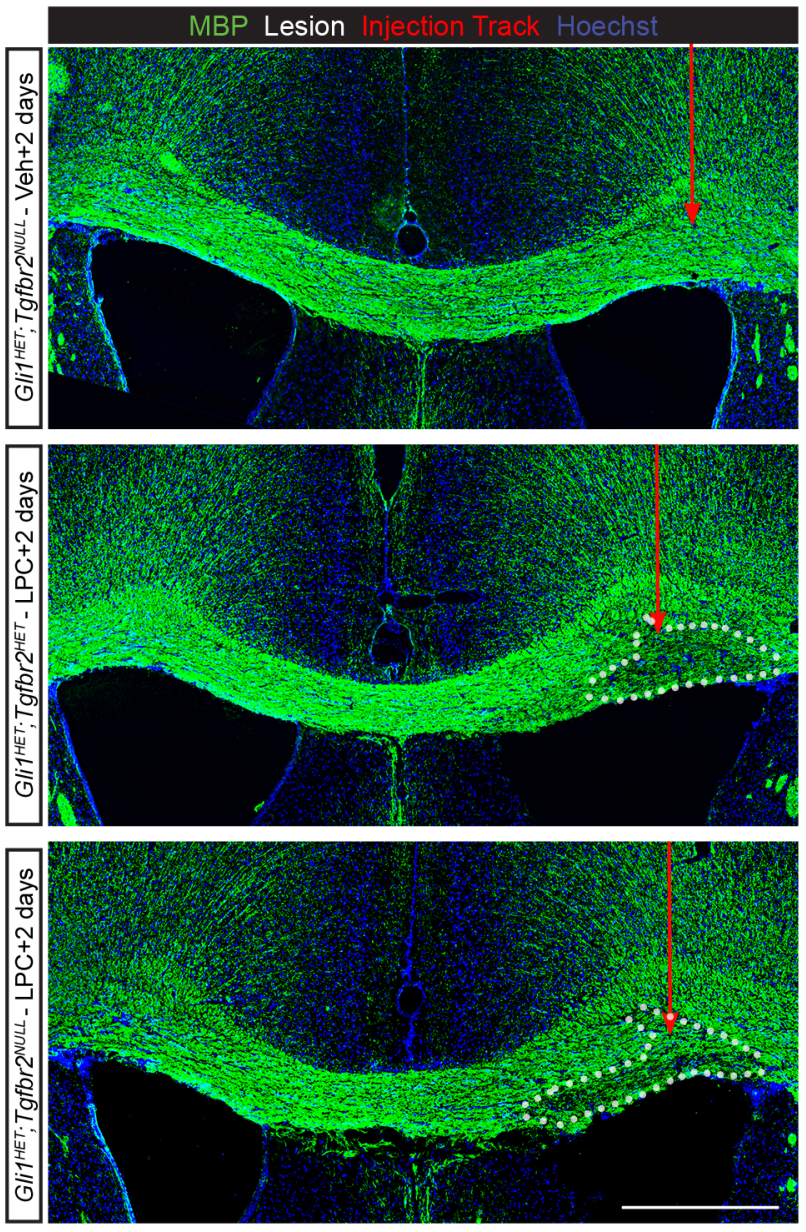


\begin{tabular}{|c|c|}
\hline Mice & $\begin{array}{l}\text { Genotype } \\
\text { (Supplier \#) }\end{array}$ \\
\hline Gli1 ${ }^{H E T}$ & $\begin{array}{c}\text { Gli1CreER/WT } \\
(\text { Jax\#007913) }\end{array}$ \\
\hline Gli1NULL & $\begin{array}{l}\text { Gli1CreER/CreER } \\
\text { (Jax\#007913) }\end{array}$ \\
\hline Gpnmb $b^{H E T}$ & $\begin{array}{c}\text { Gpnmb } \\
\text { (MMRRC\#047926-UCD) }\end{array}$ \\
\hline Gpnmb $b^{N U L L}$ & $\begin{array}{c}\text { Gpnmb Lacz/Lacz } \\
\text { (MMRRC\#047926-UCD) }\end{array}$ \\
\hline Gli1HET;Gpnmb ${ }^{H E T}$ & 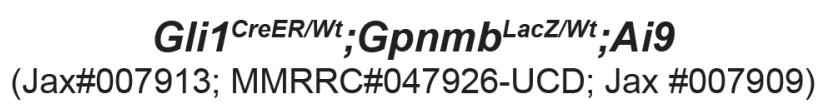 \\
\hline Gli1 $^{H E T} ; G p n m b^{N U L L}$ & 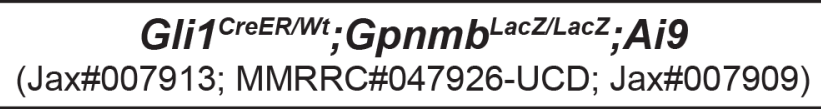 \\
\hline Gli1 ${ }^{H E T} ;$ Tgfbr $2^{H E T}$ & $\begin{array}{c}\text { Gli1CreER/Wt;Tgfbr2Flox/Wt;Ai9 } \\
\text { (Jax\#007913; Jax\#012603; Jax\#007909) }\end{array}$ \\
\hline Gli1HET;Tgfbr2 ${ }^{\text {NULL }}$ & $\begin{array}{c}\text { Gli1 }{ }^{\text {CreER/Wt } ; \text { Tgfbr2Flox/Flox } ; A i 9} \\
\text { (Jax\#007913; Jax\#012603; Jax\#007909) }\end{array}$ \\
\hline Gli1 ${ }^{N U L L} ; T g f b r 2^{H E T}$ & $\begin{array}{c}\text { Gli1CreER/CreER;Tgfbr2Flox/Wt;Ai9 } \\
\text { (Jax\#007913; Jax\#012603; Jax\#007909) }\end{array}$ \\
\hline Gli1 ${ }^{N U L L ; T g f b r 2^{N U L L}}$ & $\begin{array}{c}\text { Gli1CreER/CreER;Tgfbr2Flox/Flox;Ai9 } \\
\text { (Jax\#007913; Jax\#012603; Jax\#007909) }\end{array}$ \\
\hline
\end{tabular}

